Evaluation of Full Reynolds Stress Turbulence Models in FUN3D for Axisymmetric Jet Flow

Julianne C. Dudek

Glenn Research Center, Cleveland, Ohio 


\section{NASA STI Program . . . in Profile}

Since its founding, NASA has been dedicated to the advancement of aeronautics and space science. The NASA Scientific and Technical Information (STI) Program plays a key part in helping NASA maintain this important role.

The NASA STI Program operates under the auspices of the Agency Chief Information Officer. It collects, organizes, provides for archiving, and disseminates NASA's STI. The NASA STI Program provides access to the NASA Technical Report Server-Registered (NTRS Reg) and NASA Technical Report ServerPublic (NTRS) thus providing one of the largest collections of aeronautical and space science STI in the world. Results are published in both non-NASA channels and by NASA in the NASA STI Report Series, which includes the following report types:

- TECHNICAL PUBLICATION. Reports of completed research or a major significant phase of research that present the results of NASA programs and include extensive data or theoretical analysis. Includes compilations of significant scientific and technical data and information deemed to be of continuing reference value. NASA counter-part of peer-reviewed formal professional papers, but has less stringent limitations on manuscript length and extent of graphic presentations.

- TECHNICAL MEMORANDUM. Scientific and technical findings that are preliminary or of specialized interest, e.g., "quick-release" reports, working papers, and bibliographies that contain minimal annotation. Does not contain extensive analysis.
- CONTRACTOR REPORT. Scientific and technical findings by NASA-sponsored contractors and grantees.

- CONFERENCE PUBLICATION. Collected papers from scientific and technical conferences, symposia, seminars, or other meetings sponsored or co-sponsored by NASA.

- SPECIAL PUBLICATION. Scientific, technical, or historical information from NASA programs, projects, and missions, often concerned with subjects having substantial public interest.

- TECHNICAL TRANSLATION. Englishlanguage translations of foreign scientific and technical material pertinent to NASA's mission.

For more information about the NASA STI program, see the following:

- Access the NASA STI program home page at http://www.sti.nasa.gov

- E-mail your question to help@sti.nasa.gov

- Fax your question to the NASA STI Information Desk at 757-864-6500

- Telephone the NASA STI Information Desk at 757-864-9658

- Write to:

NASA STI Program

Mail Stop 148

NASA Langley Research Center Hampton, VA 23681-2199 


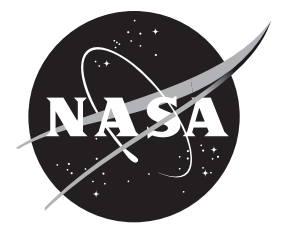

\section{Evaluation of Full Reynolds Stress Turbulence Models in FUN3D for Axisymmetric Jet Flow}

Julianne C. Dudek

Glenn Research Center, Cleveland, Ohio

Prepared for

2019 SciTech Forum

sponsored by the American Institute of Aeronautics and Astronautics

San Diego, California, January 7-11, 2019

National Aeronautics and

Space Administration

Glenn Research Center

Cleveland, Ohio 44135 


\section{Acknowledgments}

The authors would like to thank NASA's Transformational Tools and Technologies Project, Revolutionary Computational Aerosciences Subproject, managed by Mujeeb Malik, for funding this work. The authors are also grateful to Jan-Renee Carlson and Chris Rumsey, of the NASA Langley Research Center, for their assistance in using the Reynolds stress models in FUN3D, and Jim DeBonis, Nick Georgiadis, Dennis Yoder, Vance Dippold and Mary Jo Long-Davis, of the NASA Glenn Research Center, for numerous discussions and guidance regarding this work.

This report is a formal draft or working paper, intended to solicit comments and ideas from a technical peer group.

This report contains preliminary findings, subject to revision as analysis proceeds.

This work was sponsored by the Transformative Aeronautics Concepts Program.

Trade names and trademarks are used in this report for identification only. Their usage does not constitute an official endorsement, either expressed or implied, by the National Aeronautics and Space Administration.

Level of Review: This material has been technically reviewed by technical management.

Available from

NASA STI Program

Mail Stop 148

NASA Langley Research Center

Hampton, VA 23681-2199
National Technical Information Service 5285 Port Royal Road Springfield, VA 22161

703-605-6000

This report is available in electronic form at http://www.sti.nasa.gov/ and http://ntrs.nasa.gov/ 


\title{
Evaluation of Full Reynolds Stress Turbulence Models in FUN3D for Axisymmetric Jet Flow
}

\author{
Julianne C. Dudek \\ National Aeronautics and Space Administration \\ Glenn Research Center \\ Cleveland, Ohio 44135
}

\begin{abstract}
Two full seven-equation turbulence models available in the FUN3D code are evaluated for their ability to improve the computation of challenging mixing flows encountered in aerospace propulsion. These models are the SSG/LRR and Wilcox full second-moment Reynolds stress models. They solve equations for the six components of the Reynolds stress and a seventh equation for the turbulent length scale. Two standard eddy viscosity models are also evaluated for comparison, the Spalart-Allmaras (SA) one-equation model and the Menter Shear Stress Transport (SST-V) two-equation turbulence model. Flow through an axisymmetric reference nozzle is examined at three flow conditions: subsonic unheated, subsonic heated, and near sonic unheated. Centerline profiles of velocity and turbulent kinetic energy and radial profiles of velocity, turbulent kinetic energy and turbulent stresses are examined. Results showed that the SA model did well at predicting the jet potential core length, but over-mixed the downstream flow, whereas the SST-V model over-predicted the potential core length. The Wilcox-model significantly over-predicted the potential core length and under-predicted the mixing and was not well-suited for the jet flows evaluated, however the SSG/LRR Reynolds stress model did well at predicting the mixing rate and mean velocity for all cases examined.
\end{abstract}

\section{Nomenclature}

$\tilde{a}_{i j} \quad$ Reynolds-stress anisotropy tensor

$b \quad$ mixing layer thickness

$C_{i}, C_{i}^{*} \quad$ coefficients of the Speziale-Sarkar-Gatski model

$\hat{C}_{i} \quad$ coefficients of the Wilcox RSM pressure-strain model

$C_{\mu} \quad$ equilibrium parameter

$D \quad$ Reynolds stress diffusion coefficient

$D_{i j} \quad$ specific diffusion tensor

$d \quad$ distance to nearest wall point

$F_{1} \quad$ Menter's blending function

$\tilde{k} \quad$ specific turbulent kinetic energy

$M_{a} \quad$ Acoustic Mach number, $M_{a}=u_{j e t} / a_{r e f}$

$M_{j e t} \quad$ jet Mach number, $M_{j e t}=u_{j e t} / a_{j e t}$

$\mathcal{M}_{i j} \quad$ turbulent mass flux tensor

$P_{i j} \quad$ specific turbulence production tensor

$p \quad$ static pressure

$r \quad$ radial coordinate

$S_{i j} \quad$ strain rate tensor 


$\begin{array}{ll}S_{i j}^{*} & \text { traceless strain rate tensor } \\ t & \text { time } \\ u & \text { velocity component in the } x \text {-direction } \\ U & \text { local mean streamwise velocity } \\ \Delta \tilde{u} & \text { freestream velocity difference, } \Delta \tilde{u}=\tilde{u}_{1}-\tilde{u}_{2} \\ u_{i} & \text { velocity component } \\ W_{i j} & \text { rotation tensor } \\ x & \text { spatial coordinate in the streamwise or axial direction } \\ x_{i} & \text { Cartesian coordinates } \\ y & \text { spatial coordinate in the vertical or wall-normal direction } \\ \alpha & \text { coefficient of } \omega \text { production for Wilcox RSM } \\ \hat{\alpha} & \text { closure coefficient for Wilcox RSM } \\ \alpha \omega & \text { coefficient of } \omega \text { production for SSG/LRR RSM } \\ \beta & \text { coefficient of } \omega \text { destruction for Wilcox RSM } \\ \hat{\beta} & \text { closure coefficient for Wilcox RSM } \\ \beta_{o} & \text { closure coefficient for Wilcox RSM } \\ \beta_{\omega} & \text { coefficient of } \omega \text { destruction for Wilcox RSM } \\ \hat{\gamma} & \text { closure coefficient for Wilcox RSM } \\ \delta_{i j} & \text { Kronecker delta } \\ \varepsilon_{i j} & \text { turbulent dissipation rate } \\ \zeta & \text { argument to } F_{1} \text { blending function } \\ \mu & \text { dynamic viscosity } \\ \Pi_{i j} & \text { specific pressure-strain correlation tensor } \\ \rho & \text { density } \\ \sigma & \text { coefficient of } \omega \text { diffusion for Wilcox RSM } \\ \sigma^{*} & \text { closure coefficient for Wilcox RSM } \\ \sigma_{d} & \text { coefficient of cross-diffusion for SSG/LRR RSM } \\ \sigma_{\omega} & \text { coefficient of } \omega \text { diffusion for SSG/LRR RSM } \\ \tau_{i j} & \text { viscous stress tensor } \\ \phi^{(\varepsilon)} & \text { coefficient of } \varepsilon \text { equation } \\ \phi^{(\omega)} & \text { coefficient of } \omega \text { equation } \\ \omega & \text { specific dissipation rate } \\ & \end{array}$

\section{Accents}

$\phi^{\prime \prime} \quad$ fluctuating component, $\phi^{\prime \prime}=\phi-\tilde{\phi}$

$\bar{\phi} \quad$ Reynolds averaged component, $\bar{\phi}=\lim _{\Delta t \rightarrow \infty} \frac{1}{\Delta t} \int_{t_{0}}^{t_{0}+\Delta t} \phi d t$

$\tilde{\phi} \quad$ Favre averaged component, $\tilde{\phi}=\frac{1}{\bar{\rho}} \lim _{\Delta t \rightarrow \infty} \frac{1}{\Delta t} \int_{t_{0}}^{t_{0}+\Delta t} \rho \phi d t$

\section{Subscripts}

$T \quad$ turbulent

$t$ total

$0 \quad$ stagnation condition

$\infty$ freestream condition 


\subsection{Introduction}

For aerospace propulsion flows, the most common practice in computational fluid dynamics (CFD) analyses is to use Reynolds-averaged Navier-Stokes (RANS) codes with one- and two-equation turbulence models. Current turbulence models predict steady, fully turbulent attached flows at all speed regimes reasonably well, but are still unable to reliably predict jet mixing flows. Large-eddy simulation (LES) and direct-numerical simulation (DNS) methods are being used for some applications, however they require very fine grids for wall bounded flows and shear layers and at high Reynolds numbers, and therefore will not be practical for many years (Refs. 1 and 2). Hybrid RANS/LES methods are increasingly common for certain classes of simulations, although techniques to combine the near-wall RANS region with the outer, large-eddy simulation region need further development (Ref. 1).

For aircraft exhaust nozzles, RANS solvers have been used heavily and have been successful at calculating performance quantities such as thrust, but have had less success at calculating the correct mixing and turbulent structures in the jet plume. Reduction of noise produced by jets is a major focus of the aerospace industry, and to contribute to noise reduction efforts, CFD calculations must be able to correctly calculate the turbulence quantities needed as input to acoustic solvers.

RANS will be used for a significant portion of CFD analyses for the foreseeable future, due to the limitations in computational power required for LES and related techniques (Ref. 1). Traditional RANS linear and nonlinear one- and two-equation turbulence models are frequently used. The Spalart-Allmaras (SA) (Ref. 3) and Menter Shear-Stress Transport (SST) (Ref. 4) formulations are linear models which incorporate the Boussinesq approximation to give the Reynolds shear stress tensor in terms of the mean strain rate tensor and the eddy viscosity (Ref. 5). Nonlinear models incorporate additional higher-order terms that are functions of the mean strain and rotation rate tensors. An alternative to these approaches is to use a more advanced form of RANS turbulence modeling, where the individual transport equations for each of the Reynolds stresses are solved, allowing for a more detailed representation of the flow physics. These models are known as full second-moment Reynolds Stress Models (RSMs).

This paper describes a study using the FUN3D unstructured CFD code to evaluate two RSMs for flow through an axisymmetric nozzle at three conditions. The two RSMs evaluated are the combined SpezialeSarkar-Gatski/Launder-Reece-Rodi (SSG/LRR) RSM (Ref. 6) and the Wilcox RSM (Ref. 5). This study continues the work of Reference 7, which examined the performance of the RSMs for shock-boundary layer interactions and mixing flows and was performed in support of the NASA Revolutionary Computational Aerosciences (RCA) technical challenge to: "Identify and down-select critical turbulence, transition, and numerical method technologies for $40 \%$ reduction in predictive error against standard test cases for turbulent separated flows, evolution of free shear flows and shock-boundary layer interactions on state-of-the-art high performance computing hardware. "(Ref. 1). The current study examines axisymmetric nozzle flows which are part of a consensus data set compiled by Bridges and Wernet (Ref. 8) for the acoustic reference nozzle (ARN) and are also designated as RCA challenge cases. The three flow conditions examined are: set point (SP) 3 at acoustic Mach number 0.5 unheated, SP 23 at acoustic Mach number 0.5 heated, and SP7 at acoustic Mach number 0.9 unheated. Results were compared with the SA and SST-V models, and with experimental data.

\subsection{The FUN3D Code}

The FUN3D (Ref. 9) code was used for the computations described herein. It was developed by researchers at the NASA Langley Research Center and solves the RANS equations using a node-centered, unstructured implicit solver, finite-volume discretization and is formally second-order accurate in space. Explicit terms are calculated using Roe's flux difference splitting, however other methods are available. 
More information about FUN3D can be found at fun3d.larc.nasa.gov and in Reference 9. Note that at the time of this study, the full Reynolds stress models in FUN3D cannot be used for periodic grids whose side planes are rotated through a small angle. Instead, it requires $90^{\circ}$ grids with both axes aligned with one of the constant $x$-, $y$ - and $z$-coordinate surfaces. For the SA and SST-V models, small-angle periodic boundary conditions are available, so grids one-cell wide in the circumferential direction were used.

The Roe second order upwind scheme was used for the calculations. All cases were first run in steady state mode, but if convergence could not be reached, they were run using a second-order optimized timeaccurate method, as noted.

\subsection{The Turbulence Models}

The full RSM turbulence models evaluated in this work are the combined Speziale-SarkarGatski/Launder-Reece-Rodi (SSG/LRR) RSM and the Wilcox RSM. The more standard Spalart-Allmaras (SA) one-equation model (Ref. 3) and the Menter shear stress transport (SST-V) two-equation model (Ref. 4) were also used for comparison with the RSMs. Note that the "SST-V" model is the particular form of the SST model that uses vorticity for the turbulent production source term. Details about all of these models are available on the Turbulence Modeling Resource (TMR) website, turbmodels.larc.nasa.gov (Ref. 10). The SA and SST-V models use the Boussinesq approximation,

$$
\bar{\rho} \tau_{i j}=2 \mu_{T}\left(\tilde{S}_{i j}-\frac{1}{3} \tilde{S}_{k k} \delta_{i j}\right)-\frac{2}{3} \rho k \delta_{i j}
$$

a constitutive relation, to compute the turbulent stress tensor, $\tau_{i j}$, defined below as,

$$
\tau_{i j} \stackrel{\text { def }}{=} \overline{-u_{i}^{\prime \prime} u_{j}^{\prime \prime}}
$$

On the other hand, the RSMs solve transport equations for each of the six unique Reynolds stresses. A seventh equation is required to determine the turbulent length scale variable.

The SSG/LRR full Reynolds stress model, as described on the TMR website, and in References 6 and 11, was developed under the European Union project FLOMANIA and consists of the six equations of the Reynolds stress transport equations, plus Menter's baseline $\omega$ equation for the length scale. The Reynolds-stress transport equation is given by Equation (3).

$$
\frac{\partial\left(\bar{\rho} \tau_{i j}\right)}{\partial t}+\frac{\partial\left(\bar{\rho} \tau_{i j} \tilde{u}_{k}\right)}{\partial x_{k}}=-\bar{\rho} P_{i j}-\bar{\rho} \Pi_{i j}+\bar{\rho} \varepsilon_{i j}-\bar{\rho} D_{i j}-\bar{\rho} \mathcal{M}_{i j}
$$

The pressure-strain model, given by Equation (4), blends the Speziale-Sarkar-Gatski model with the LaunderReece-Rodi model near the wall, as defined in References 6 and 10.

$$
\begin{aligned}
\Pi_{i j} & =-\left(C_{1} \varepsilon+\frac{1}{2} C_{1}^{*} P_{k k}\right) \tilde{a}_{i j}+C_{2} \varepsilon\left(\tilde{a}_{i k} \tilde{a}_{k j}-\frac{1}{3} \tilde{a}_{k l} \tilde{a}_{k l} \delta_{i j}\right)+\left(C_{3}-C_{3}^{*} \sqrt{\tilde{a}_{k l} \tilde{a}_{k l}}\right) \tilde{k} \tilde{S}_{i j}^{*} \\
& +C_{4} \tilde{k}\left(\tilde{a}_{i k} \tilde{S}_{j k}+\tilde{a}_{j k} \tilde{S}_{i k}-\frac{2}{3} \tilde{a}_{k l} \tilde{S}_{k l} \delta_{i j}\right)+C_{5} \tilde{k}\left(\tilde{a}_{i k} \widetilde{W}_{j k}+\tilde{a}_{j k} \widetilde{W}_{i k}\right)
\end{aligned}
$$

The remaining terms in Equation (3) are given below.

$$
P_{i j}=\tau_{i k} \frac{\partial \tilde{u}_{j}}{\partial x_{k}}+\tau_{j k} \frac{\partial \tilde{u}_{i}}{\partial x_{k}} \quad \text { Production }
$$




$$
\begin{array}{ll}
\bar{\rho} \varepsilon_{i j}=\frac{2}{3} \bar{\rho} \delta_{i j} \varepsilon & \text { Dissipation } \\
\tilde{a}_{i j}=-\frac{\tau_{i j}}{\tilde{k}}-\frac{2}{3} \delta_{i j} & \text { Aniosotropy Tensor } \\
\tilde{S}_{i j}=\frac{1}{2}\left(\frac{\partial \tilde{u}_{i}}{\partial x_{j}}+\frac{\partial \tilde{u}_{j}}{\partial x_{i}}\right) & \text { Strain Rate Tensor } \\
\tilde{S}_{i j}^{*}=\tilde{S}_{i j}-\frac{1}{3} \tilde{S}_{k k} \delta_{i j} & \text { Traceless Strain Rate Tensor } \\
\widetilde{W}_{i j}=\frac{1}{2}\left(\frac{\partial \tilde{u}_{i}}{\partial x_{j}}-\frac{\partial \tilde{u}_{j}}{\partial x_{i}}\right) & \text { Averaged Rotation Tensor } \\
\bar{\rho} D_{i j}=-\frac{\partial}{\partial x_{k}}\left[\left(\bar{\mu} \delta_{k l}-D \frac{\bar{\rho} \tau_{k l} \tilde{k}}{\varepsilon}\right) \frac{\partial\left(\tau_{i j}\right)}{\partial x_{l}}\right] & \text { Diffusion }
\end{array}
$$

The turbulent mass flux term, $\mathcal{M}_{i j}$, is assumed to be negligible.

A length scale equation is required to close the system. The SSG/LRR model uses Menter's baseline $\omega$-equation which blends an $\omega$ equation near the wall with an $\varepsilon$ equation in the outer boundary layer.

$$
\frac{\partial(\bar{\rho} \omega)}{\partial t}+\frac{\partial\left(\bar{\rho} \omega \widetilde{u}_{k}\right)}{\partial x_{k}}=\alpha_{\omega} \frac{\omega}{\tilde{k}} \frac{\bar{\rho} P_{k k}}{2}-\beta_{\omega} \bar{\rho} \omega^{2}+\frac{\partial}{\partial x_{k}}\left[\left(\bar{\mu}+\sigma_{\omega} \frac{\bar{\rho} \tilde{k}}{\omega}\right) \frac{\partial \omega}{\partial x_{k}}\right]+\sigma_{d} \frac{\bar{\rho}}{\omega} \max \left(\frac{\partial \tilde{k}}{\partial x_{k}} \frac{\partial \omega}{\partial x_{k}}, 0\right)
$$

The isotropic dissipation rate is defined as

$$
\varepsilon=C_{\mu} \tilde{k} \omega
$$

with $C_{\mu}=0.09$. The equations used for blending the coefficients $\phi=\alpha_{\omega}, \beta_{\omega}, \sigma_{\omega}, \sigma_{d}$ are given below.

$$
\begin{gathered}
\phi=F_{1} \phi^{(\omega)}+\left(1-F_{1}\right) \phi^{(\varepsilon)} \\
F_{1}=\tanh \left(\zeta^{4}\right) \\
\zeta=\min \left[\max \left(\frac{\sqrt{\tilde{k}}}{C_{\mu} \omega d}, \frac{500 \mu}{\bar{\rho} \omega d^{2}}\right), \frac{4 \sigma_{\omega}^{(\varepsilon)} \bar{\rho} \tilde{k}}{\sigma_{d}^{(\varepsilon)} \frac{\bar{\rho}}{\omega} \max \left(\frac{\partial \tilde{\kappa} \partial \omega}{\partial x_{k} \partial x_{k}}, 0\right) d^{2}}\right]
\end{gathered}
$$

where $d$ is the distance to the nearest wall. The pressure-strain coefficients are blended between LaunderReece-Rodi (LRR) (Ref. 12) near walls (without wall-correction terms) and Speziale-Sarkar-Gatski (SSG) (Ref. 13) away from walls. The coefficients are given in Table I.

TABLE I.-COEFFICIENTS FOR THE SSG/LRR FULL REYNOLDS STRESS TURBULENCE MODEL

\begin{tabular}{|c|c|c|c|c|c|c|c|c|c|c|c|c|}
\hline & $\alpha_{\omega}$ & $\beta_{\omega}$ & $\sigma_{\omega}$ & $\sigma_{d}$ & $C_{1}$ & $C_{1}^{*}$ & $C_{2}$ & $C_{3}$ & $C_{3}^{*}$ & $C_{4}$ & $C_{5}$ & $D$ \\
\hline $\mathrm{LRR}^{(\omega)}$ & 0.5556 & 0.075 & 0.5 & 0 & 1.8 & 0 & 0 & 0.8 & 0 & $\frac{\left(9 C_{2}^{L R R}+6\right)}{11}$ & $\left(-7 C_{2}^{L R R} 10\right)$ & 11 \\
\hline $\mathrm{SSG}^{(\varepsilon)}$ & 0.44 & 0.0828 & 0.856 & 1.712 & 1.7 & 0.9 & 1.05 & 0.8 & 0.65 & 0.625 & 0.2 & 0.22 \\
\hline
\end{tabular}


The diffusion term given in Equation (11), is a generalized gradient diffusion model (Ref. 14). In the FUN3D input file, the SSG/LRR RSM is specified as "SSG/LRR-RSM-w2012." There is also a version of the SSG/LRR RSM which uses a simple diffusion model (Ref. 15) and is specified as "SSG/LRRRSM-w2012-SD" in the input file. In this model, the diffusion term is modeled as given in Equation (17)

$$
\bar{\rho} D_{i j}=-\frac{\partial}{\partial x_{k}}\left[\left(\bar{\mu}-\frac{D}{C_{\mu}} \mu_{T}\right) \frac{\partial\left(\tau_{i j}\right)}{\partial x_{k}}\right] \quad \text { Simple Diffusion }
$$

with:

$$
D=0.5 C_{\mu} F_{1}+\frac{2}{3} 0.22\left(1-F_{1}\right)
$$

In this paper, the simple diffusion model, which tends to be more stable, was used. In addition, the correct implementation of the SSG/LRR RSM in FUN3D was verified by comparisons with results from two independently developed flow solvers on a sequence of successively refined grids. Results give a high level of confidence that the model is implemented correctly (Ref. 16).

The Wilcox RSM as described on the TMR website and in Reference 5 also solves the six equations for the Reynolds stress tensor and an omega equation for the specific dissipation rate, $\omega$. The Reynolds stress equation is given by Equation (19).

$$
\frac{\partial\left(\bar{\rho} \tau_{i j}\right)}{\partial t}+\frac{\partial\left(\bar{\rho} \tau_{i j} \widetilde{u}_{k}\right)}{\partial x_{k}}=-\bar{\rho} P_{i j}-\bar{\rho} \Pi_{i j}+\frac{2}{3} \beta^{*} \bar{\rho} \omega k \delta_{i, j}+\frac{\partial}{\partial x_{k}}\left[\left(\bar{\mu}+\sigma^{*}\right) \frac{\partial \tau_{i j}}{\partial x_{k}}\right]
$$

The specific dissipation rate is given by Equation (20).

$$
\frac{\partial \bar{\rho} \omega}{\partial t}+\frac{\partial\left(\bar{\rho} \omega \widetilde{u}_{j}\right)}{\partial x_{j}}=\alpha \frac{\bar{\rho} \omega}{k} \tau_{i j} \frac{\partial \widetilde{u}_{i}}{\partial x_{j}}-\beta \bar{\rho} \omega^{2}+\sigma_{d} \frac{\bar{\rho}}{\omega} \frac{\partial k}{\partial x_{j}} \frac{\partial \omega}{\partial x_{j}}+\frac{\partial}{\partial x_{k}}\left[\left(\bar{\mu}+\sigma \mu_{T}\right) \frac{\partial \omega}{\partial x_{k}}\right]
$$

The pressure strain correlation is given by:

$$
\Pi_{i j}=\beta^{*} \hat{C}_{1} \omega\left(\tau_{i j}+\frac{2}{3} k \delta_{i j}\right)-\hat{\alpha}\left(P_{i j}-\frac{2}{3} P \delta_{i j}\right)-\hat{\beta}\left(D_{i j}-\frac{2}{3} P \delta_{i j}\right)-\hat{\gamma} k\left(S_{i j}-\frac{1}{3} S_{k k} \delta_{i j}\right)
$$

with,

$$
P=\frac{1}{2} P_{k k} \quad \mu_{T}=\bar{\rho} k / \omega \quad D_{i j}=\tau_{i k} \frac{\partial \tilde{u}_{k}}{\partial x_{j}}+\tau_{j k} \frac{\partial \tilde{u}_{k}}{\partial x_{i}}
$$

The production, $P_{i j}$, given by Equation (5), the simple strain rate tensor, $S_{i j}$, given by Equation (8), and the averaged rotation tensor, $\widetilde{W}_{i j}$, given by Equation (10), are used in both the SSG/LRR and Wilcox RSMs. The closure coefficients for Equation (21) are given in Table II, and in Equations (23) and (24).

$$
\begin{gathered}
\sigma_{d}=\left\{\begin{array}{l}
0, \frac{\partial k}{\partial x_{j}} \frac{\partial \omega}{\partial x_{j}} \leq 0 \\
\frac{1}{8}, \frac{\partial k}{\partial x_{j}} \frac{\partial \omega}{\partial x_{j}}>0
\end{array}\right. \\
f_{\beta}=\frac{1+85 \mathrm{X}_{\omega}}{1+100 \mathrm{X}_{\omega}} \quad \mathrm{X}_{\omega}=\left|\frac{W_{i j} W_{j k} \hat{S}_{k i}}{\left(\beta^{*} \omega\right)^{3}}\right| \quad \hat{S}_{k i}=S_{k i}-\frac{1}{2} \frac{\partial \bar{u}_{m}}{\partial x_{m}} \delta_{k, i}
\end{gathered}
$$


TABLE II.-CLOSURE COEEFICIENTS FOR THE WILCOX FULL REYNOLDS-STRESS TURBULENCE MODEL

\begin{tabular}{|c|c|c|c|c|c|c|c|c|c|c|}
\hline$\hat{\alpha}$ & $\hat{\beta}$ & $\hat{\gamma}$ & $\hat{C}_{1}$ & $\hat{C}_{2}$ & $\alpha$ & $\beta$ & $\beta^{*}$ & $\sigma$ & $\sigma^{*}$ & $\beta_{o}$ \\
\hline$\left(8+C_{2}\right) / 11$ & $\left(8-C_{2}\right) / 11$ & $\left(60 C_{2}-4\right) / 55$ & $\frac{9}{5}$ & $\frac{10}{19}$ & $\frac{13}{25}$ & $\beta_{o} f_{\beta}$ & $\frac{9}{100}$ & 0.5 & 0.6 & 0.0708 \\
\hline
\end{tabular}

TABLE III.-DEFINITION OF TEST CONDITIONS

\begin{tabular}{|c|c|c|c|c|c|}
\hline Set point & $M_{a}$ & $T_{j e t} / T_{\infty}$ & NPR & $M_{j e t}$ & $T_{t} / T_{\infty}$ \\
\hline 3 & 0.500 & 0.95 & 1.197 & 0.513 & 1.000 \\
\hline 23 & 0.500 & 1.764 & 1.102 & 0.376 & 1.814 \\
\hline 7 & 0.900 & 0.835 & 1.861 & 0.985 & 1.000 \\
\hline
\end{tabular}

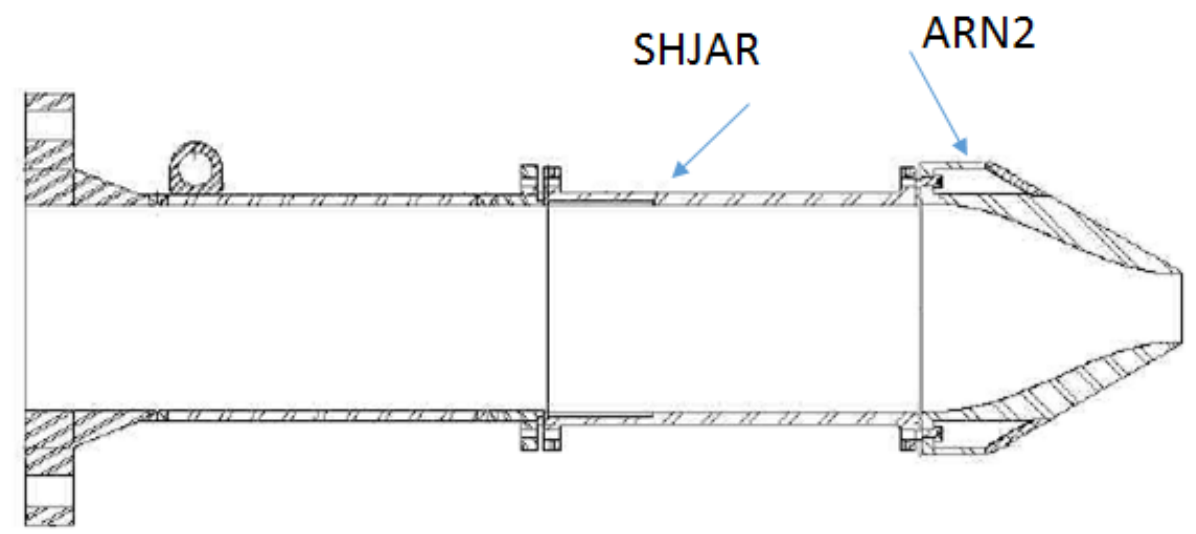

Figure 1.-SHJAR nozzle system with Acoustic Research Nozzle 2 (ARN2) attached (Ref. 8).

\subsection{Experimental Configuration}

A consensus data set for a range of axisymmetric jet flows was created by Bridges and Wernet at the NASA Glenn Research Center (Ref. 8). It is a compilation of data from 15 sources of particle image velocimetry (PIV) testing in the Small Hot Jet Acoustic test Rig (SHJAR) from 2001 through 2007. The data contains mean and variance data for seven sets of defined flow conditions, or "set points" (SP). Of those, three set points were evaluated in this study and are given in Table III. They consisted of an unheated jet with acoustic Mach number $M_{a}=0.5$ (SP 3), a heated jet with $M_{a}=0.5$ (SP 23), and an unheated jet with $M_{a}=0.9$ (SP 7), where the acoustic Mach number is defined as $M_{a}=u_{j e t} / a_{\infty}$. In each experiment, air is passed through an axisymmetric convergent nozzle to produce a desired jet Mach number $\left(M_{j e t}=u_{j e t} / a_{j e t}\right)$ exiting into quiescent air at $P_{\infty}=14.366 \mathrm{psi}, T_{\infty}=515.7 \mathrm{R}$. The nozzle is referred to as acoustic research nozzle 2 (ARN2), and has an inlet diameter of 6.0 in., a lip thickness of $0.05 \mathrm{in}$., an outside face angle of $30^{\circ}$ to the jet axis, a parallel flow section at the exit of $0.25 \mathrm{in}$. and a nozzle exit diameter of 2.0 in. A schematic of ARN2 installed in the SHJAR is shown in Figure 1.

\subsection{CFD Methodology}

These three axisymmetric jet flow cases are described on the TMR website (Ref. 10), where results are shown for computations made using the SA and SST-V models and two CFD codes, Wind-US (Refs. 17 and 18) and CFL3D (Ref. 19). For the current study, the FUN3D (version 12.9) (Ref. 9) code was used with the SSG/LRR and Wilcox RSMs, as well as SA and SST-V models. (The FUN3D results computed using the SA and SST-V turbulence models are in good agreement with the Wind-US and CFL3D results, verifying correct setup and usage of FUN3D.) The initial and boundary conditions were 
set up to match those on the TMR. The flow external to the nozzle was set to $M_{\infty}=0.01$, since quiescent air is often difficult to compute with CFD. The external nozzle flow conditions, using this freestream Mach number, were also used as the "reference" conditions in the FUN3D input file, with reference Reynolds number equal to 5601 (based on the nozzle radius of $1 \mathrm{in}$.), and reference temperature equal to $530 \mathrm{R}$. For the boundary conditions, at the jet inflow, the nozzle pressure ratio (NPR), $p_{t} / p_{\infty}$, and the temperature ratio $T_{t} / T_{\infty}$ were specified as in Table 3 for each of the set point conditions. The nozzle walls had adiabatic viscous boundary conditions and the freestream static pressure was specified at the far downstream boundary. A far-field condition was used at the outermost radial surface boundary and symmetry conditions were used at the symmetry planes on the constant-theta boundaries.

A series of grids was available on the TMR website, each coarse grid was created by removing every other grid point from the next finest grid. The grids contained 3 zones each as shown in Figure 2 and Table IV. To convert these grids to FUN3D-compatible format, Pointwise (Ref. 20) was used, resulting in a single zone grid. For computations using the eddy viscosity models, the grids were one-cell deep spanning approximately $1^{\circ}$ in the theta direction. For the calculations using the RSMs, a $90^{\circ}$ sector grid was required, since small angle periodic boundary conditions were not available for the RSMs. To generate this grid, the axisymmetric grid was extruded $90^{\circ}$ in the theta direction at $5^{\circ}$ increments for a total of 19 grid points. Grid resolution was examined for the set point 3 flow conditions using FUN3D with the SA and SST-V turbulence models. The solution did not show appreciable changes when using Grid 129 and Grid 257, so Grid 129 was used for the remainder of the computations. Attempts were made to use Grid 257 with the RSMs, but a steady solution was not achieved, nor were converged time-accurate solutions.

TABLE IV.-AXISYMMETRIC JET, GRID STUDY

\begin{tabular}{|l|c|c|c|}
\hline \multicolumn{1}{|c|}{ Name } & Upstream external region & Internal nozzle region & Jet mixing region \\
\hline Grid 65 & $25 \times 25$ & $16 \times 25$ & $65 \times 57$ \\
\hline Grid 129 & $49 \times 49$ & $31 \times 49$ & $129 \times 113$ \\
\hline Grid 257 & $97 \times 97$ & $61 \times 97$ & $257 \times 225$ \\
\hline
\end{tabular}

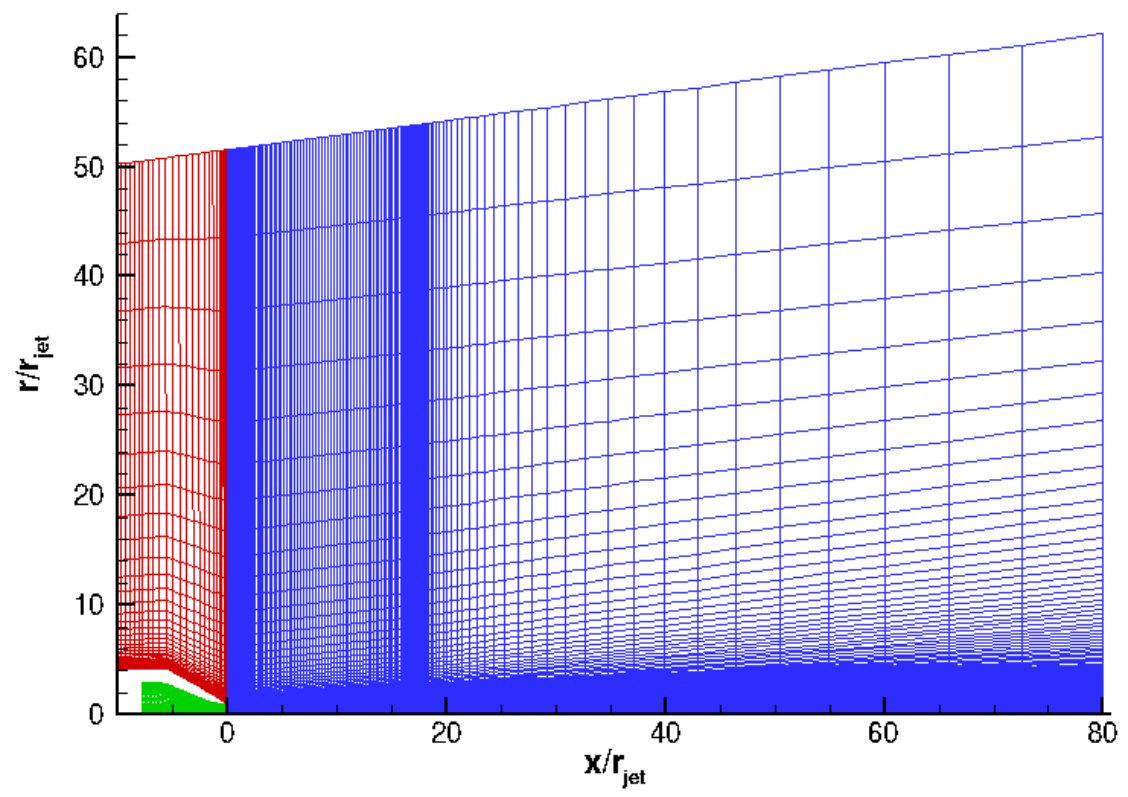

Figure 2.-Grid shown at the second from the finest level (grid 129). 
Turbulent flow exiting a jet is naturally unstable, making these calculations somewhat challenging to perform with nearly quiescent ambient conditions. For all cases, we attempted to obtain a steady converged solution, where convergence was achieved when the flow residuals had dropped significantly and the flow properties of interest were not changing after continued iterations. Set point 7, which had unheated, nearly sonic flow, was the easiest to run in terms of convergence and stability. Steady solutions were obtained for set point 7 using all four turbulence models. The SA model was the most robust turbulence model, and steady solutions were obtained for all three sets of flow conditions. A steady solution was also obtained for set point 3 using the SSG/LRR RSM. For remaining calculations, convergence was not achieved using a steady state (constant CFL number) calculation, so an optimized second order backward differencing timeaccurate method (Ref. 21) was used. Solutions were allowed to run several hundred flow-through cycles to ensure the flowfield was established. The solutions run using the RSMs required more iterations for the flow to set up as well. They also required more computational time per iteration than the eddy viscosity models, since more equations were being solved and more grid points were used on the $90^{\circ}$ grid, as well as more overall iterations to reach a converged or quasi-steady solution.

For the majority of the runs, an inviscid flux limiting scheme was used to help the solutions converge, or to improve the convergence. The limiter used was the Van Albada inviscid flux limiter with a heuristic pressure limiter (Ref. 22), and the limiter value was frozen after 30,000 time steps. For cases using both a RSM and the flux limiter, the radial $y$-velocity profiles at stations near the jet exit had unexpectedly high values near the centerline. It was discovered that turning off the flux limiter allowed v-velocity profiles to return to the expected behavior, although the solutions would not converge without the limiter for the following three cases: SP 3 with the Wilcox RSM and SP 23 using both RSMs. It was suggested (Ref. 23) that using the newer version of FUN3D, v13.2 (Ref. 24), which has a nonreflecting outflow pressure boundary condition, may allow the solutions to converge without a limiter. This proved true for set SP 7 , however the limiter was still required to achieve a converged solution for SP 3 with the Wilcox RSM and for SP 23 with both RSMs, despite the non-reflective outflow condition. The $y$-velocity profiles reflect this effect (see Figure 5 and Figure 10). The limiter did not adversely affect the radial profiles of the cases run using the SA and SST-V models, nor did it cause any noticeable changes to any of the flow quantities plotted. These changes to the $y$-velocity profiles when using the limiter with the RSMs may indicate that the flux limiter interacts with the RSM boundary conditions, however the exact cause of the problem has not been identified at the time of publication.

\subsection{Results}

The results are given in Figure 3 to Figure 17 and include centerline profiles of velocity and turbulent kinetic energy, and radial profiles at 5 locations downstream of the jet exit for streamwise and radial velocity, streamwise and radial turbulence intensity, turbulent shear stress and turbulent kinetic energy.

\subsection{Set Point 3 (Subsonic, Unheated Jet Flow)}

Results for set point 3, the subsonic, unheated case, are shown in Figure 3 to Figure 7. The mean $x$ velocity along the centerline is given in Figure 3, indicating that the length of the potential core (defined as the location where the centerline velocity is 98 percent of the jet exit value) in the experiment was 6.2 jet diameters. All of the turbulence models over-predicted the potential core length, with the SA model in closest agreement with experiment, predicting a potential core of 6.5 jet diameters, followed by the SSG/LRR RSM with 7.3 diameters. The SST-V model over-predicts the potential core length to be 8.5 jet diameters and the Wilcox RSM significantly over-predicts the core to be 11.7 jet diameters. The SSG/LRR RSM has the best mixing rate downstream of the potential core, whereas the Wilcox RSM has 

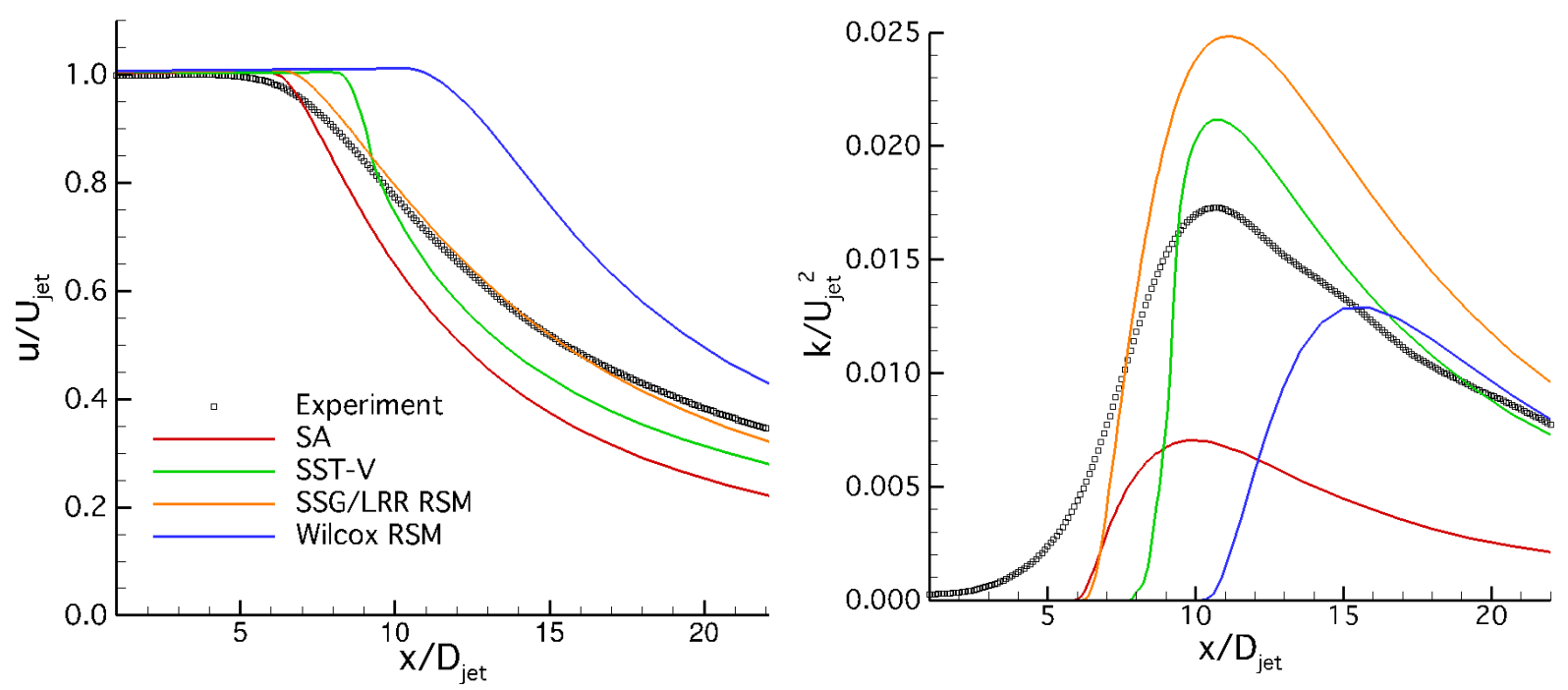

Figure 3.-Set point 3. Centerline profiles of axial velocity (left) and turbulent kinetic energy (right).

the worst agreement and is significantly under-mixed. The two eddy viscosity models show too much mixing downstream of the potential core, resulting in lower values of the centerline velocity downstream. Overall, the SSG/LRR RSM gave the best prediction of the centerline velocity and the Wilcox RSM gave the worst.

The turbulent kinetic energy, $k$, on the centerline is given in Figure 3. For the SA model, $k$ is approximated using Bradshaw's formula, where $k=\mu_{T} \sqrt{2 S_{i j} S_{i j}} /(0.31 \rho)$. For all of the turbulence models, there is significant variance from the experiment. The general profile shape resembles the experimental profile, although the values begin to increase from zero just before the end of the potential core. In the experiment, however, the values begin to increase at the nozzle exit $\left(x / D_{j e t}=0\right)$, before significant mixing begins. It has been hypothesized that a large portion of what has been measured as turbulence near $x / D_{j e t}=0$ may be oscillations in the velocity near the centerline that are more a result of the inviscid stream tube changing size due to turbulence at the shear layer edge (Ref. 25). RANS calculations are unable to compute fluctuations in the inviscid core and the turbulence models can only produce and sustain turbulence in regions containing mean velocity shear, which is not present in this region. In terms of the peak value of turbulent kinetic energy, the SST-V model is in closest agreement with the experiment, over-predicting it by 22 percent. The Wilcox RSM under-predicts the peak value by 25 percent, The SSG/LRR RSM over-predicts the peak value by 44 percent and the SA model underpredicts the peak value by 54 percent. The axial locations of the peak turbulent kinetic energy are all close to the experiment, with the exception of the Wilcox RSM, which has peak value about 5 jet exit diameters downstream of the experimental peak location. The end of the potential core is near the location of the onset of the rise of the turbulent kinetic energy, other than that, there are no obvious relationships between the two quantities in terms of agreement with the experimental data.

Radial velocity profiles at $x / D_{\text {jet }}=2,5,10,15$, and 20 are given in Figure 4 . Near the jet exit, the Wilcox RSM result shows a slightly thicker potential core, as indicated by the jet velocity beginning to mix with the ambient air at a slightly greater distance from the centerline. Beginning at $x / D_{j e t}=10$, and further downstream, the SA result shows slightly more decay of the jet near the centerline, and the Wilcox RSM shows less decay. At $x / D_{j e t}=15$ and 20, the SST-V model result also shows too much decay near the centerline. The SSG/LRR result is in good agreement with the experimental $x$-velocity profiles at all axial stations examined. The radial $y$-velocity profiles are shown in Figure 5. With the exception of the Wilcox RSM, all of the turbulence model results are in similar agreement with the experimental data in 
the potential core and shear layer. (Recall from Section 5.0 that a flux limiter was required to achieve a converged solution for the Wilcox RSM, and it was learned that the limiter adversely effects the transverse velocity profiles.) Outside of the shear layer, the SSG/LRR RSM is in best agreement with ambient region. Radial profiles of the turbulent kinetic energy are given in Figure 6. Near the jet exit at $x / D_{\text {jet }}=2$, all of the turbulence model results have profile shapes similar to the data, as expected, with the Wilcox RSM peak value closest to the experiment, however, the potential core is thicker and the shear layer is thinner than the data and the other turbulence model results. The SA model computes the highest peak $k$ value in the potential core at $x / D_{j e t}=2$, and the SST-V and SSG/LRR models have similar peak values and profiles at $x / D_{\text {jet }}=2,5$, and 10 . Further downstream, the SST-V model is in best agreement with the experiment.

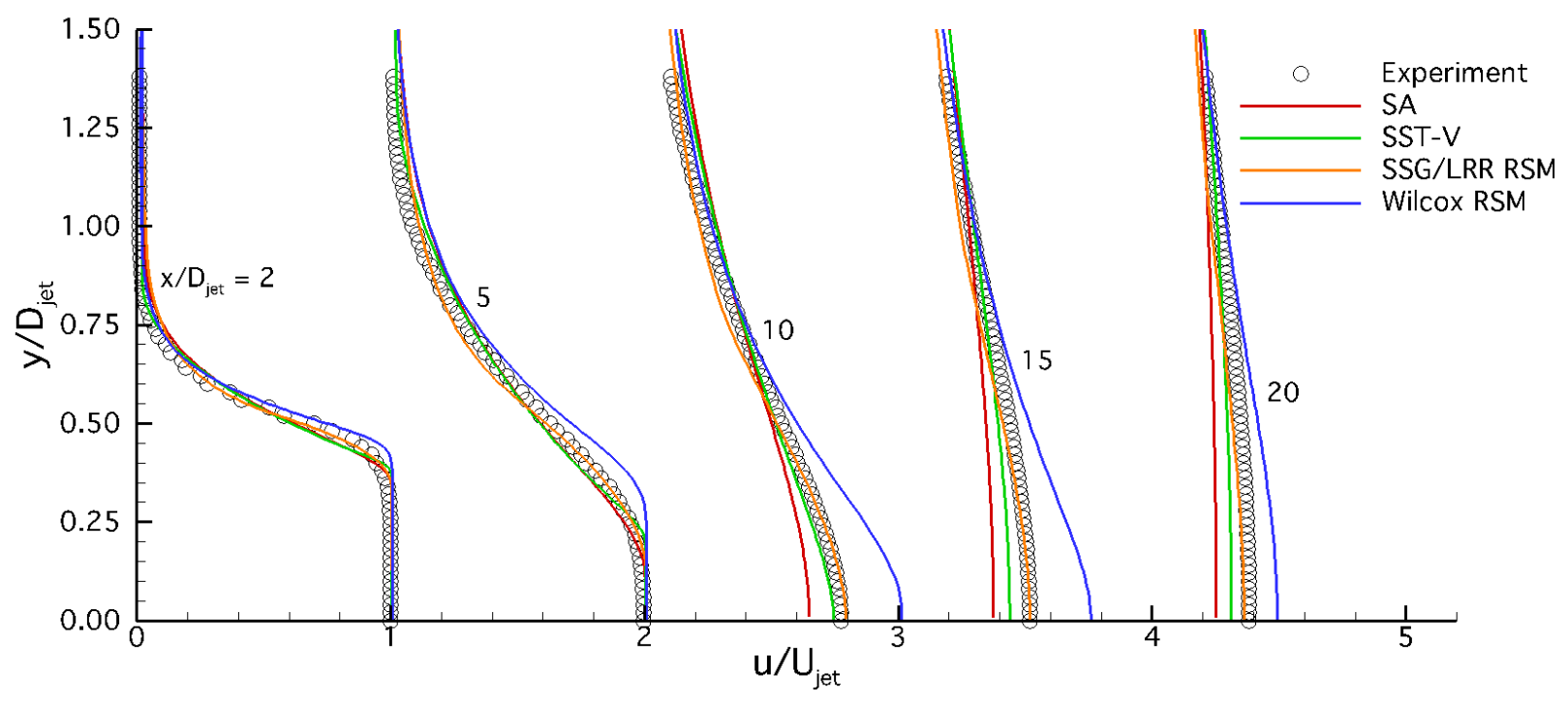

Figure 4.-Set point 3. Radial $x$-velocity profiles. (Subsequent profiles shifted by $u / \cup_{j e t}=1.0$ )

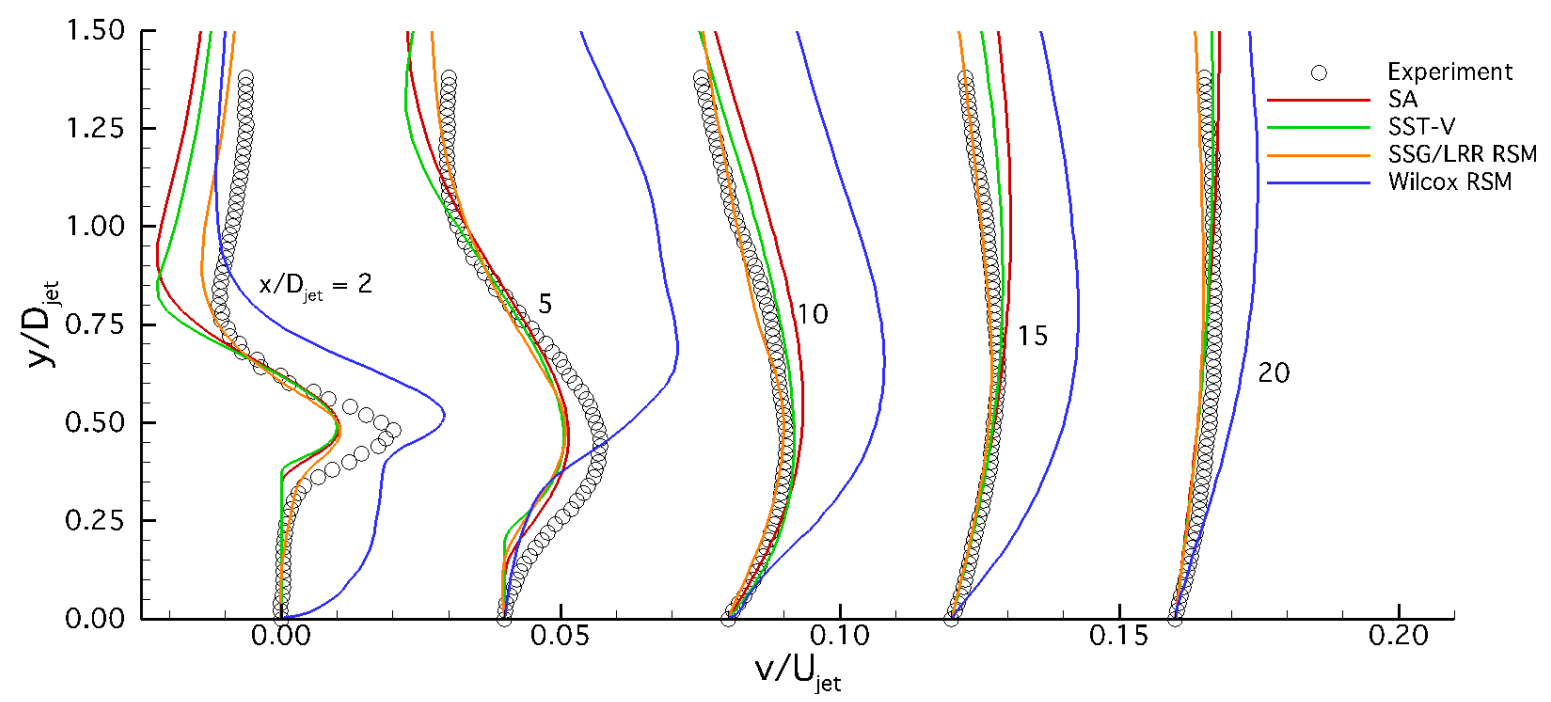

Figure 5.-Set point 3. Radial $y$-velocity profiles. (Subsequent profiles shifted by $u / U_{j e t}=0.04$ ) 


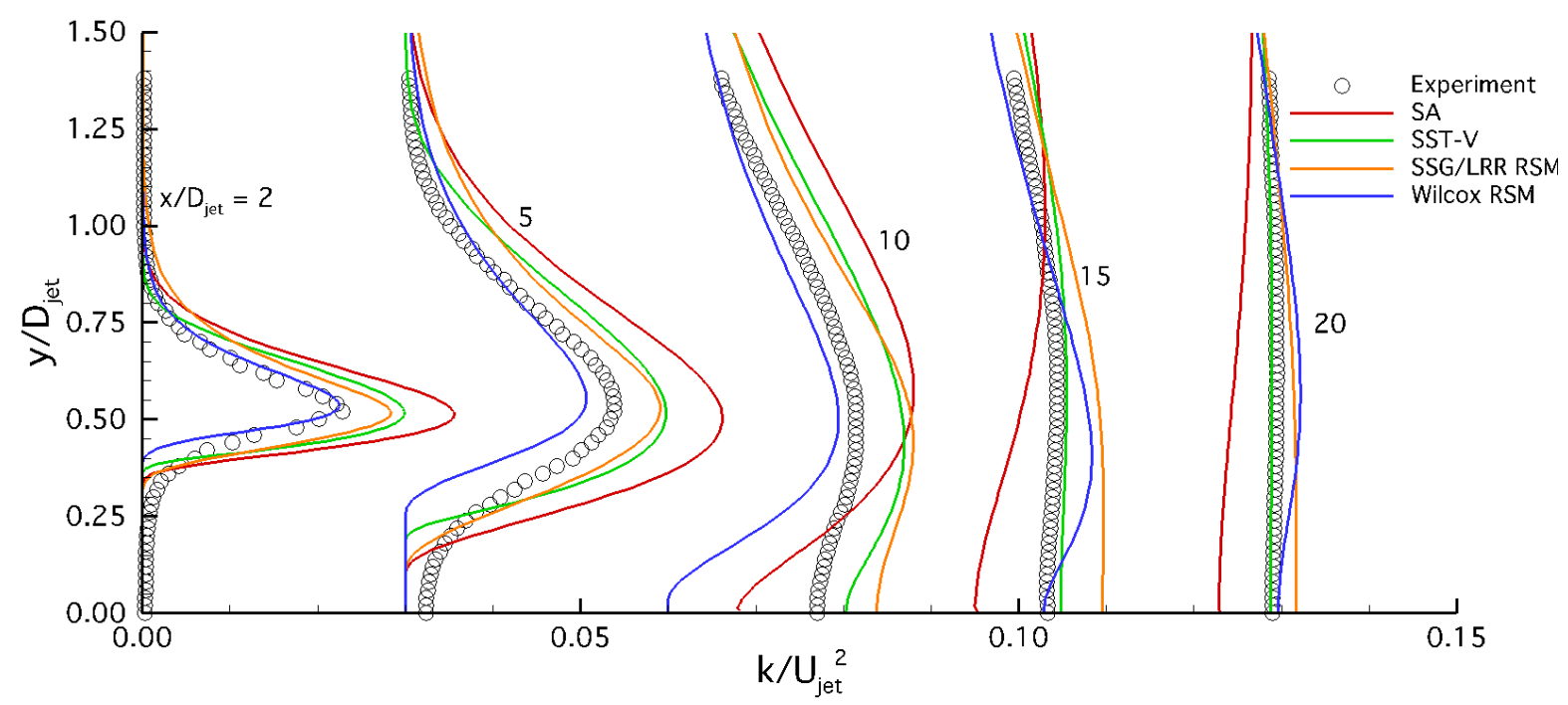

Figure 6.-Set point 3. Radial profiles of turbulent kinetic energy. (Subsequent profiles shifted by $k / \cup_{j e t}{ }^{2}=0.03$ )

Radial profiles of the components of the turbulent stress are given in Figure 7. For the SA and SST-V models, $u^{\prime} u^{\prime}, v^{\prime} v^{\prime}$, and $u^{\prime} v^{\prime}$ are computed using the Boussinesq approximation (Eq. (1)) with Bradshaw's approximation used for $k$ in the SA model results. At stations closer to the jet exit, the Wilcox model results exhibit a slightly thicker potential core. For the $u^{\prime} u^{\prime}$ stress component, at $x / D_{j e t}=2$, all of the models agree reasonably well with the data in terms of peak values and general profile shape. At $x / D_{j e t}=$ $5,10,15$, and 20, the SSG/LRR RSM is in the worst agreement with the experiment, predicting high values. The SST-V results are in the best agreement with data, which is fortuitous, and should not be the case, since the SST-V model assumes isotropic turbulence $\left(u^{\prime} u^{\prime}=v^{\prime} v^{\prime}=w^{\prime} w^{\prime}\right)$. For the $v^{\prime} v^{\prime}$ component of turbulent stress, the SSG/LRR RSM is in good agreement with the data at axial stations, and the Wilcox RSM is in reasonably close agreement. The eddy viscosity models predict high values for $v^{\prime} v^{\prime}$ at the upstream stations, and SST-V agrees well with the data at $x / D_{\text {jet }}=20$. For the $u^{\prime} v^{\prime}$ turbulent shear stress, near the jet exit, the Wilcox RSM is in closest agreement with the experimental data, even though it has the worst overall mixing (Figure 3), which is tied to $u^{\prime} v^{\prime}$. The SA, SST-V and SSG/LRR RSM results are similar, predicting similar profile shapes, but higher peak values. The Wilcox RSM shows a thicker potential core at $x / D_{\text {jet }}=5$, and also at $x / D_{\text {jet }}=10$, with values near the centerline still higher than the experiment at $x / D_{j e t}=15$ and 20 . The other models have turbulent shear stress profile shapes similar to the data at $x / D_{\text {jet }}=20$.

Overall, using the SSG/LRR RSM may have advantages over the eddy viscosity models. The SSG/LRR RSM did well at predicting the centerline velocity profile and potential core length. It also did well at predicting the radial components of velocity and shear stress in the potential core. The Wilcox RSM results should be viewed with caution, since a limiter was required to reach a converged solution, and it has been found that the using a limiter effects the boundary conditions, gives suspect $y$-velocity profiles, and most likely effects the other flow quantities. The Wilcox RSM gave poor predictions of the jet mixing. 

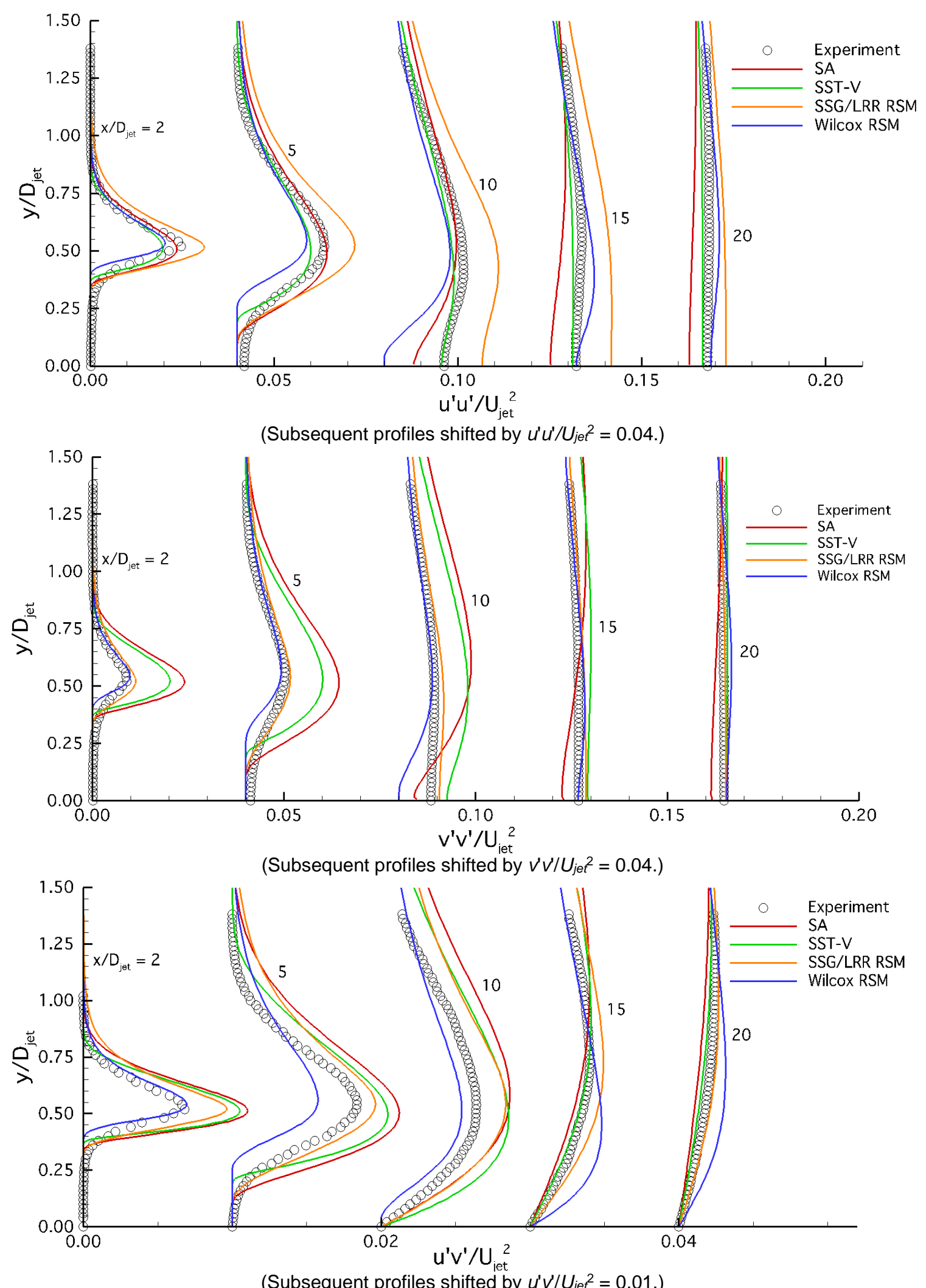

Figure 7.-Set point 3. Radial profiles of the turbulent stress components, $u^{\prime} u^{\prime}, v^{\prime} v^{\prime}$, and $u^{\prime} v^{\prime}$. 


\subsection{Set Point 23 (Subsonic, Heated Jet Flow)}

Set point 23 results are given in Figure 8 to Figure 12 . The mean $x$-velocity along the centerline is given in Figure 8. The profiles are somewhat similar to those for SP 3, although heating the incompressible jet has shortened the potential core slightly, reduced its diameter, and increased the mixing rate. In the experiment, the inviscid core length is 4.9 jet exit diameters long and is 21 percent (1.3 diameters) shorter than the unheated result. The SA model and the SSG/LRR RSM predict potential core lengths in closest agreement with the experiment, and although none of the linear models replicate the trend with heating, the RSMs are better at capturing the "shortening" trend. The SA model and the SSG/LRR RSM results give potential core lengths of 5.8 and 6.0 diameters, respectively, and have decreased in length from the unheated case by 10 and 18 percent, respectively. The SST-V and Wilcox RSM both predict noticeably longer potential core lengths of 7.3 and 9.5 diameters, respectively, and have decreased from the unheated case by 10 and 19 percent, respectively. As in the unheated case, the SA model over-mixes the flow and the Wilcox RSM under-mixes it. The SSG/LRR RSM provides good agreement with data for overall mixing, however, unlike the SP 3 result, the SST-V model also fortuitously does well mixing the downstream flow. The turbulent kinetic energy along the centerline is also shown in Figure 8. Compared to the unheated case $\left(k_{\text {peak }} / U_{\text {jet }}{ }^{2}=0.0173\right)$, the experimental peak value is slightly higher $\left(k_{\text {peak }} / U_{\text {jet }}^{2}=0.022\right)$ and 2.75 diameters closer to the jet exit at $x / D_{\text {jet }}=7.95$. The SST-V result is in closest agreement with the experimental peak value and overall profile shape; the location of the peak value has moved upstream significantly by 1.6 diameters, although the peak value has changed negligibly from the unheated case. The SA result is similar to the unheated case, and the peak value and location have negligible changes from the SP 3 values. The SSG/LRR and Wilcox RSM peak values have increased and shifted upstream slightly, exhibiting similar trends as the experimental data, and the SSG/LRR RSM peak $k$ value is also in better agreement with the data than for the SP3 result.

Radial profiles of the velocity components and the turbulent kinetic energy are given in Figure 9 to Figure 11. In the $x$-velocity profiles of Figure 9, the profiles are similar to those of SP 3 . The behavior of the turbulence models appears very similar to set point 3 . In the $y$-velocity profiles of Figure 10, the eddy viscosity models are in reasonably good agreement with the experiment, as in set point 3 , however the RSMs are both in poor agreement near the jet exit, and the Wilcox RSM result continues to disagree with the data to station $x / D_{j e t}=15$. Recall that for this case, solutions using both RSMs required a flux limiter to achieve a converged solution, and it was found that this also effects the $y$-velocity. For the radial $k$ profiles shown in Figure 11, the turbulence model behavior is very similar to the set point 3 results.
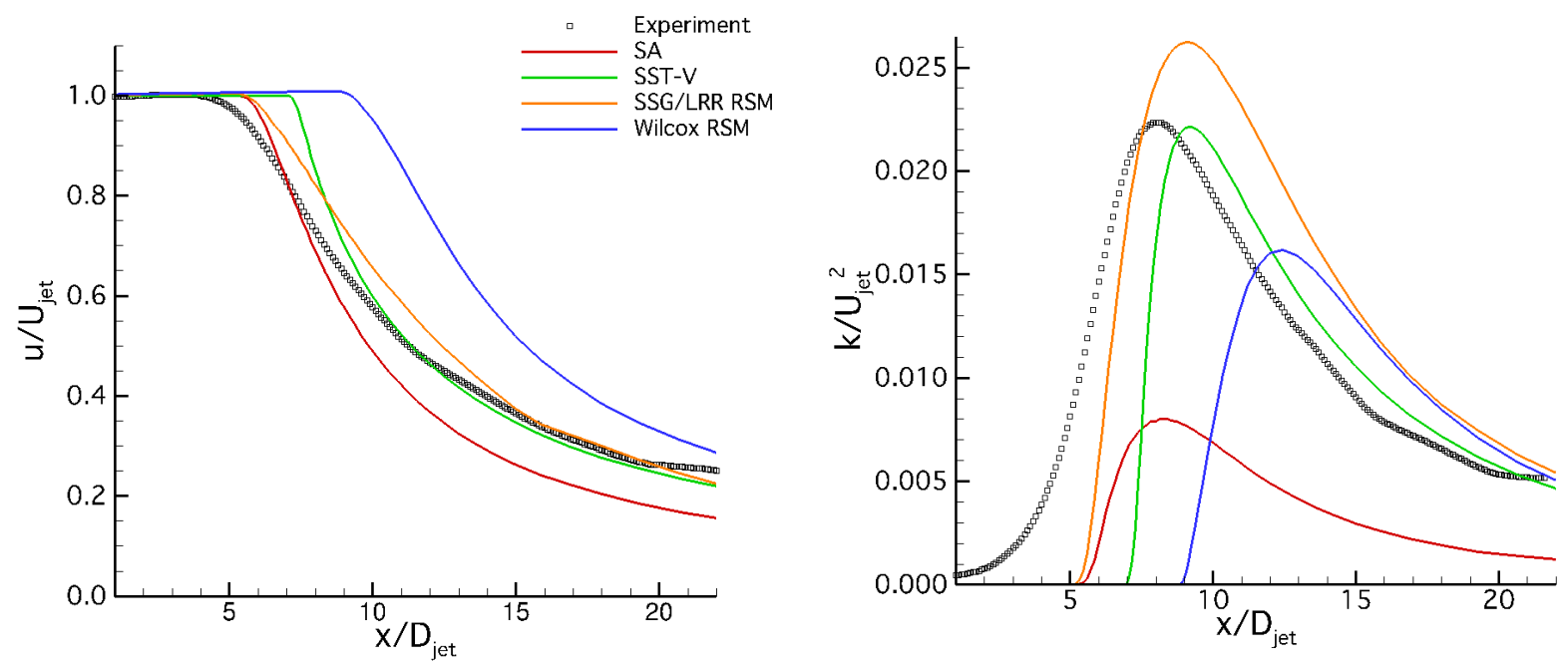

Figure 8.-Set point 23. Centerline profiles of axial velocity (left) and turbulent kinetic energy (right). 


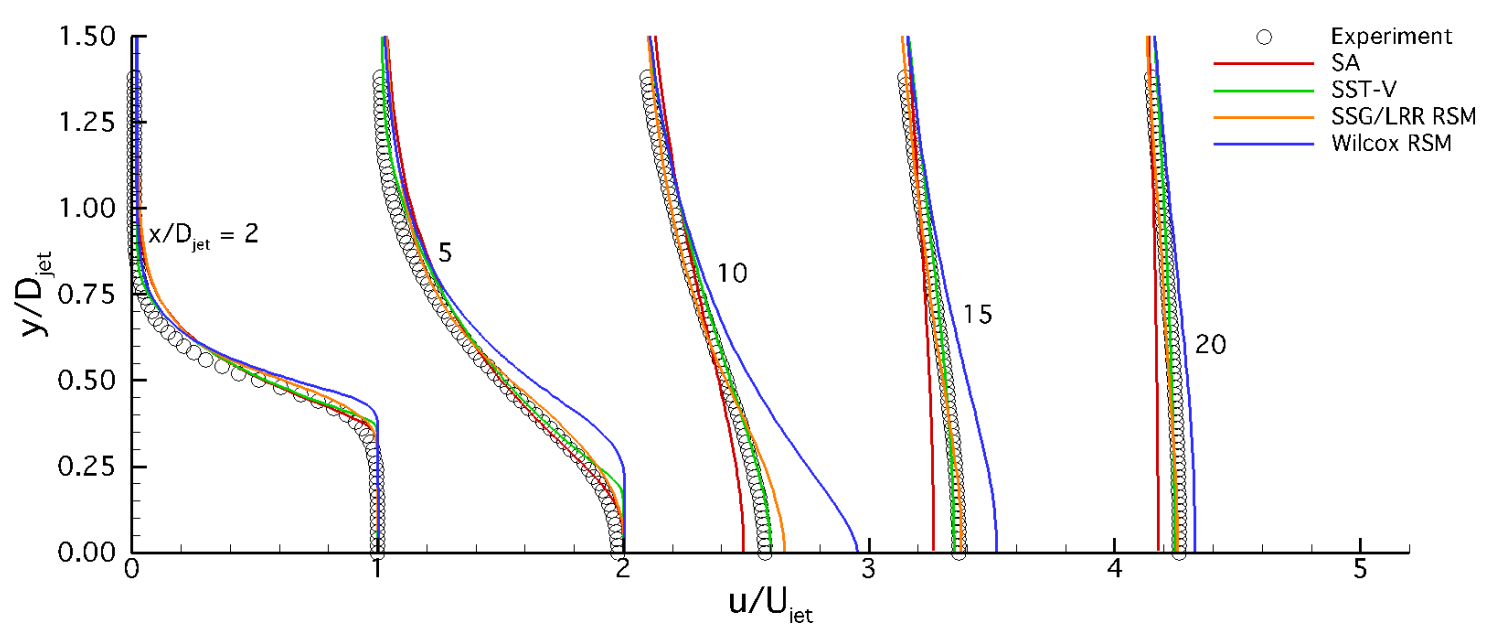

Figure 9.-Set point 23. Radial $x$-velocity profiles. (Subsequent profiles shifted by $u / \cup_{j e t}=1.0$ )

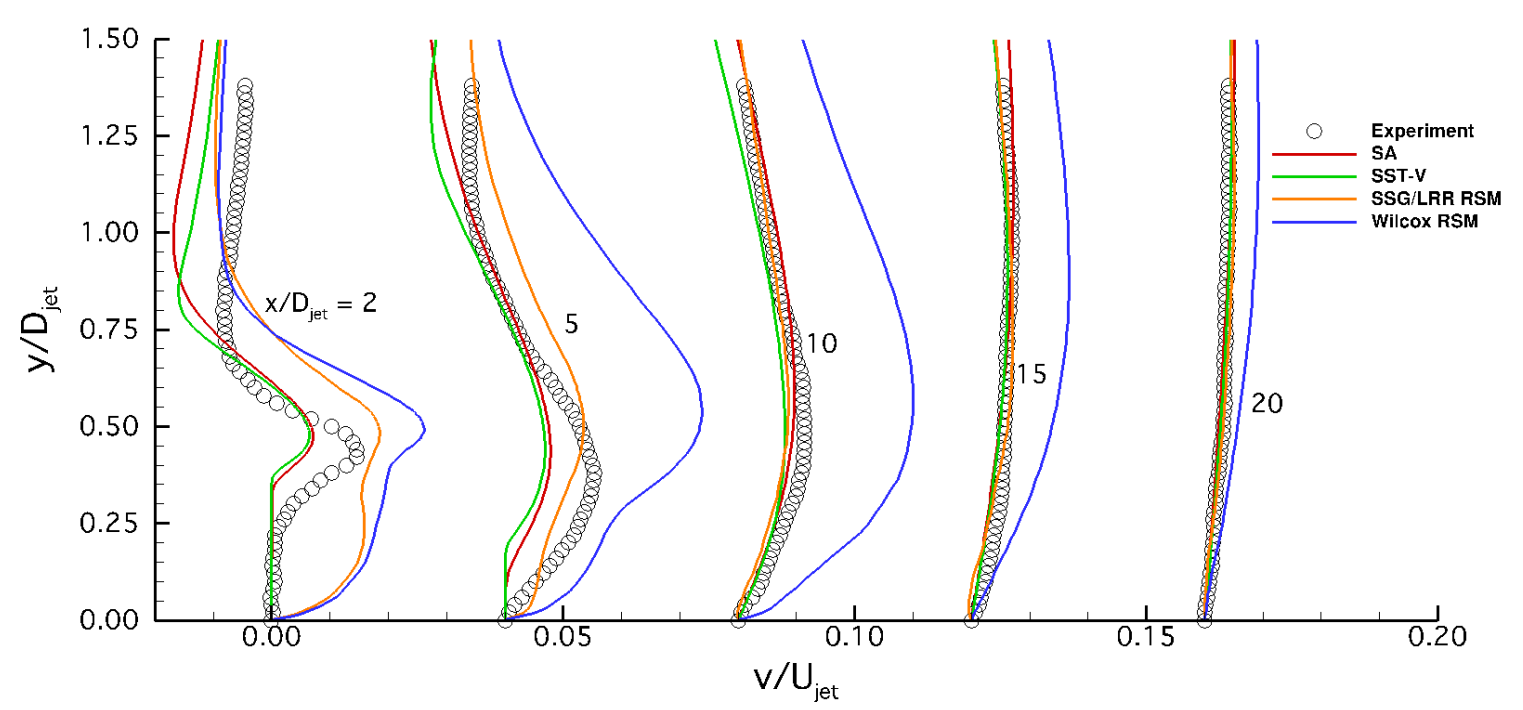

Figure 10.-Set point 23. Radial $y$-velocity profiles. (Subsequent profiles shifted by $u / \cup_{j e t}=0.04$ )

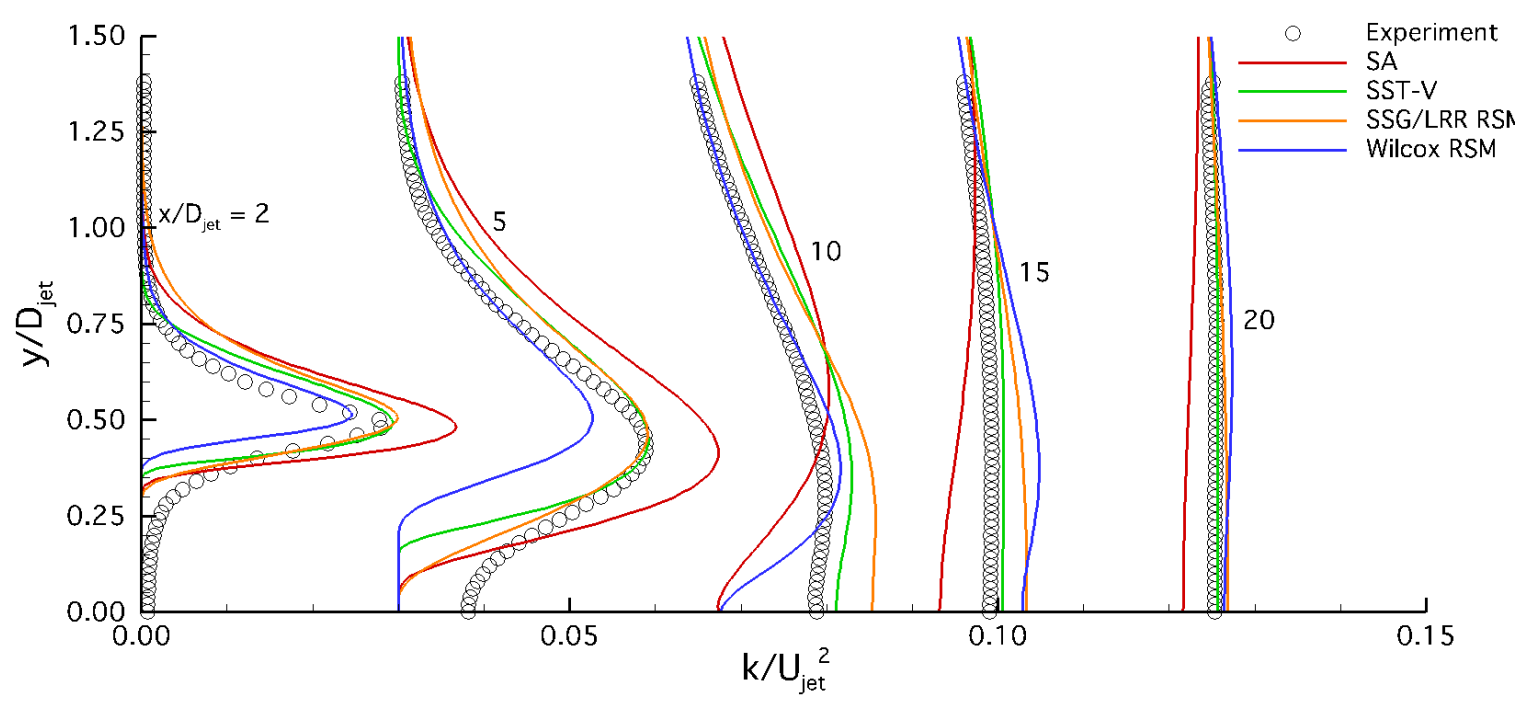

Figure 11.-Set point 23. Radial profiles of turbulent kinetic energy. (Subsequent profiles shifted by $k / U_{j e t}{ }^{2}=0.03$ ) 
Radial profiles of the components of the turbulent stresses are given in Figure 12. In general, the potential core is smaller in diameter than the SP 3 flow at similar axial stations, with slightly higher peak values, because of the more rapid mixing. Overall the performance of the turbulence models is similar to that exhibited in the SP 3 results.

\subsection{Set Point 7(Near-Sonic, Unheated Jet Flow)}

Set point 7 results are given in Figure 13 to Figure 17. The mean $x$-velocity along the centerline is shown in Figure 13. The profiles are somewhat similar to those for SP 3 (unheated, subsonic flow), although the potential core is slightly longer in length, due to compressibility. In the experiment, the inviscid core length is 7.8 jet diameters and is 1.6 diameters longer than the subsonic unheated case. The SSG/LRR RSM predicts the potential core length to be 7.2 jet diameters and is in the closest agreement with the data. The SA model under-predicts the core length to be 6.7 diameters, and the SST-V and Wilcox RSM results give potential core lengths of 9.1 and 11.9 diameters, respectively. The SA, SST-V and SSG/LRR RSM all indicate faster mixing than observed in the experiment, with the SSG/LRR RSM in overall best agreement with the data. The Wilcox RSM under-predicts the mixing. The turbulent kinetic energy along the centerline is also shown in Figure 8. The experimental peak value of $k$ increased slightly from the set point 3 value of $k_{\text {peak }} / U_{\text {jet }}{ }^{2}=0.017$ to $k_{\text {peak }} / U_{\text {jet }}{ }^{2}=0.018$, and the location of the peak value moved 2.3 jet diameters further downstream. The SST-V result is in closest agreement with the experimental peak value and the location of the peak value moved downstream slightly, although it is still approximately 2 jet diameters upstream of the experiment. The SA result is similar to the subsonic, unheated case. The Wilcox, and SSG/LRR RSM peak values are nearly the same as those for SP 3, and the location of the peaks only moved less than one half of a jet diameter downstream of the SP 3 values. It should be noted that none of the RANS approaches used any kind of compressibility correction, which are empirically designed to match the experimentally observed reduction in free shear layer mixing at higher speeds where compressibility is a factor.

Radial profiles of the velocity components and the turbulent kinetic energy are given in Figure 14 to Figure 16. In the $x$-velocity profiles of Figure 14, the profiles are similar to those of SP 3 , however near the jet exit, the potential core is slightly thicker. The behavior of the turbulence models appears very similar to SP 3. In the $y$-velocity profiles of Figure 15, all of the turbulence model results are in reasonably good agreement with the experiment. Recall that for this case, the solutions run using the RSMs did not require a limiter to reach convergence, so the problem with the limiter effecting the boundary conditions did not exhibit itself in the y-velocity profiles. For the radial $k$-profiles shown in Figure 16, the turbulence model behavior is very similar to the SP 3 results.

Radial profiles of the components of the turbulent stresses are given in Figure 17. In general, the profiles are similar to set point 3 results with respect to shape and peak values. Overall the performance of the SA, SST-V and SSG/LRR models is similar to that exhibited in the SP 3 results, and the Wilcox RSM results are in better agreement with the data near the jet exit, but not further downstream.

Overall, this case was more robust to run, required fewer iterations, and did not require the use of a limiter to reach convergence. The SSG/LRR RSM does the best job at predicting the potential core length and centerline mixing. It also did well at predicting the transverse shear stress in the potential core. 

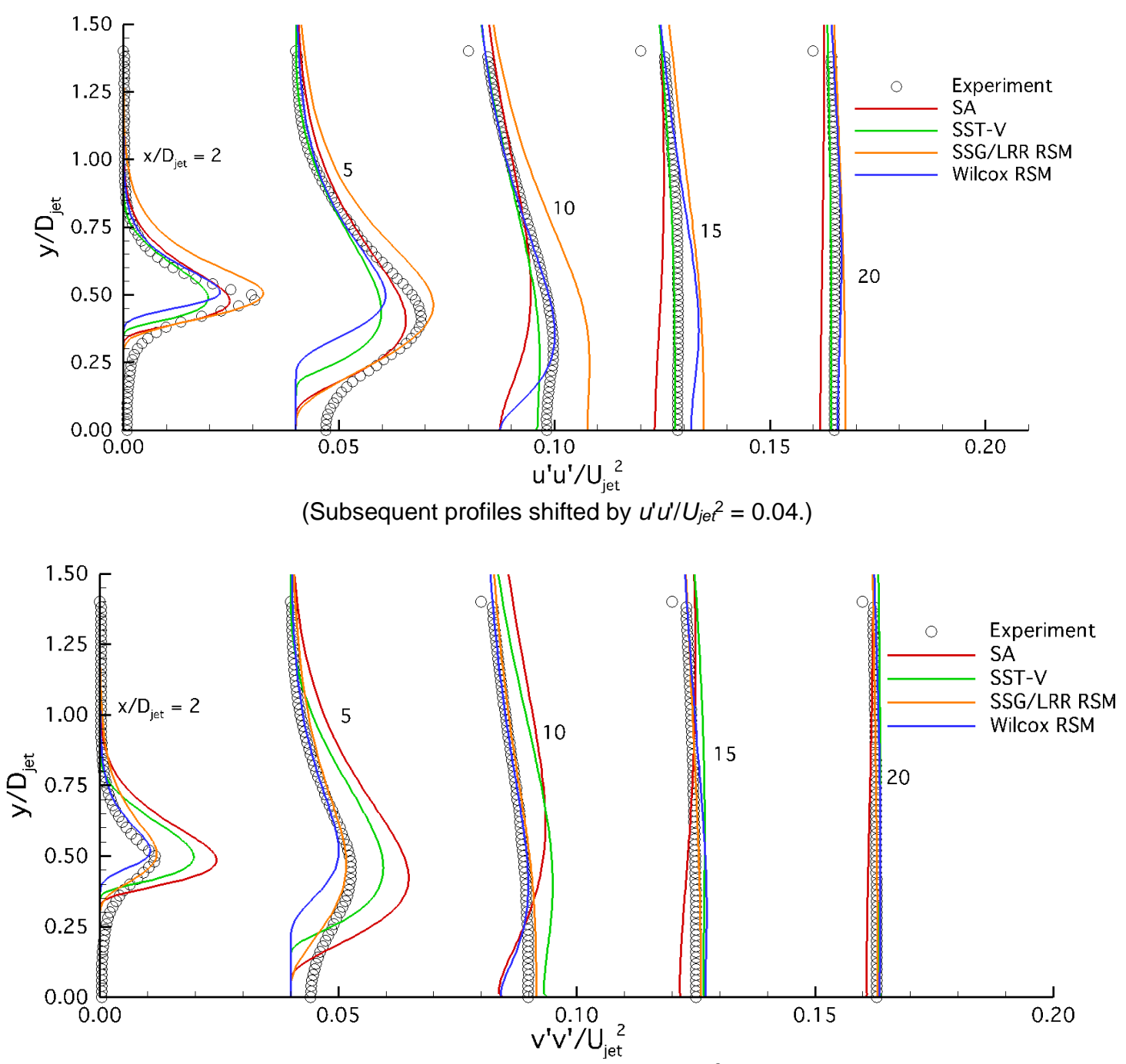

(Subsequent profiles shifted by $v^{\prime} v^{\prime} / U_{\text {jet }}^{2}=0.04$.)

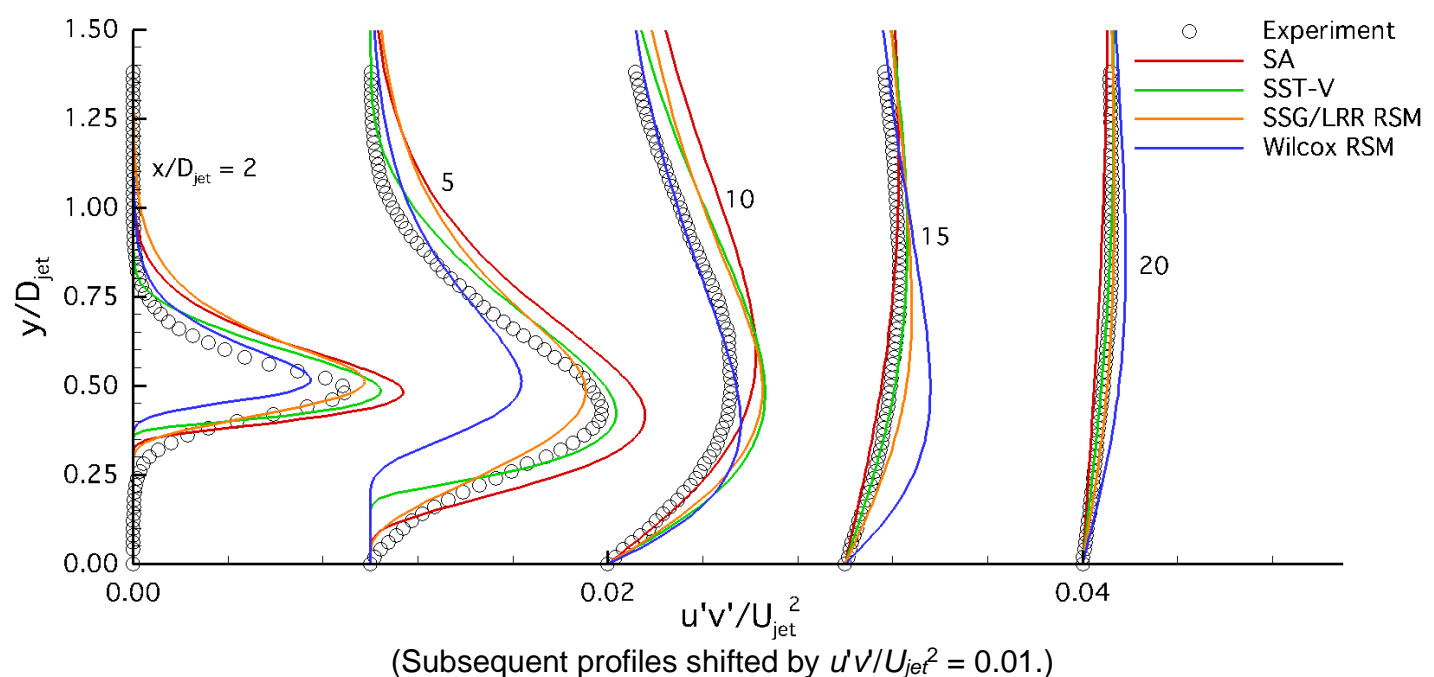

Figure 12.-Set point 23. Radial profiles of the turbulent stress components, $u^{\prime} u^{\prime}, v^{\prime} v^{\prime}$, and $u^{\prime} v^{\prime}$. 

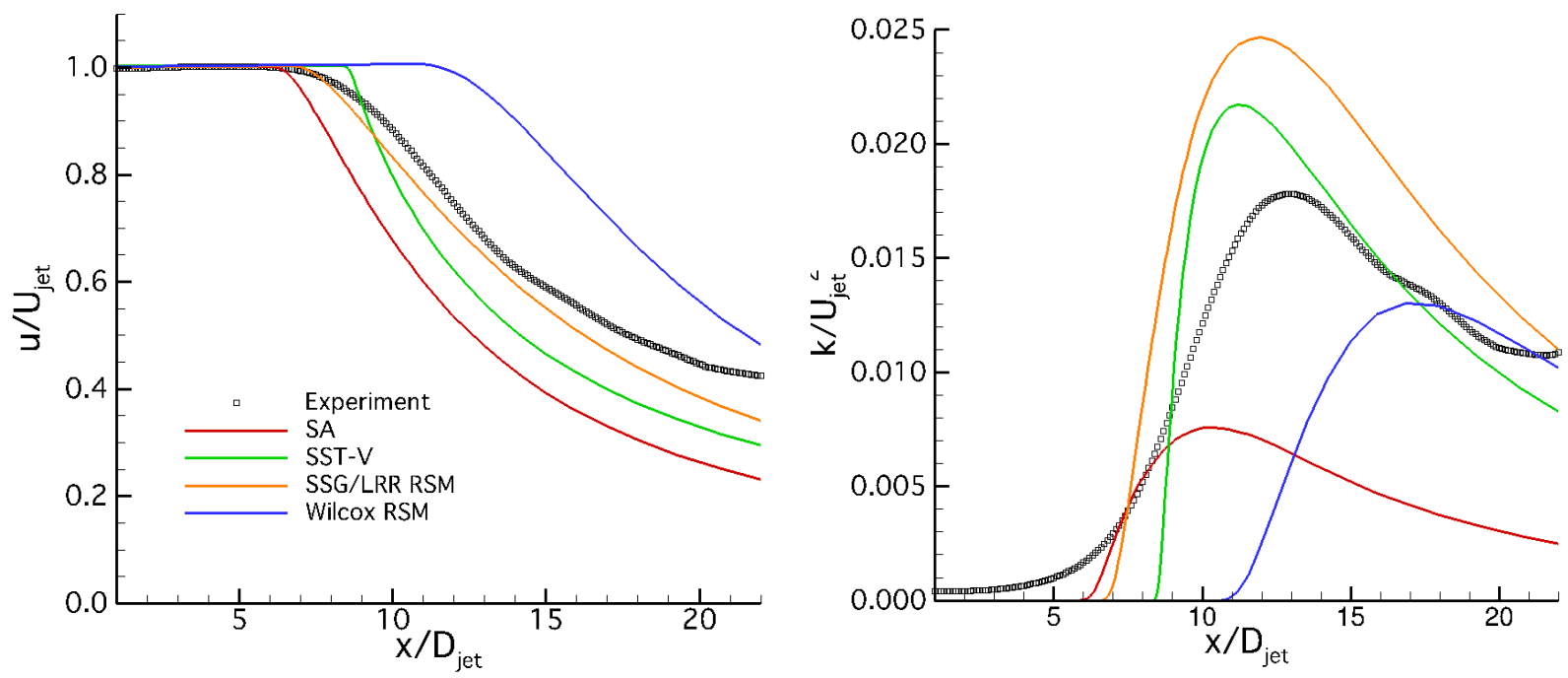

Figure 13.-Set point 7. Centerline profiles of axial velocity (left) and turbulent kinetic energy (right).

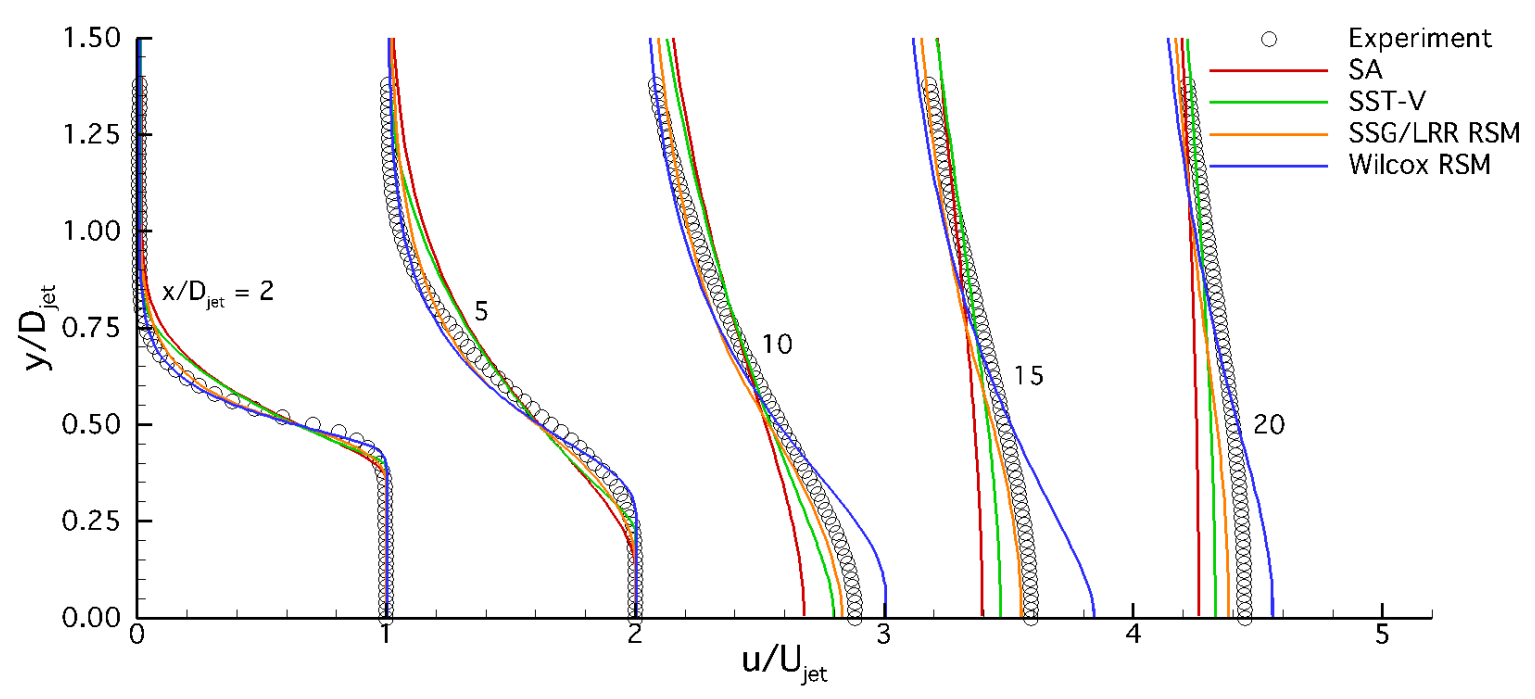

Figure 14.-Set point 7. Radial $x$-velocity profiles. (Subsequent profiles shifted by $u / U_{j e t}=1.0$ ) 


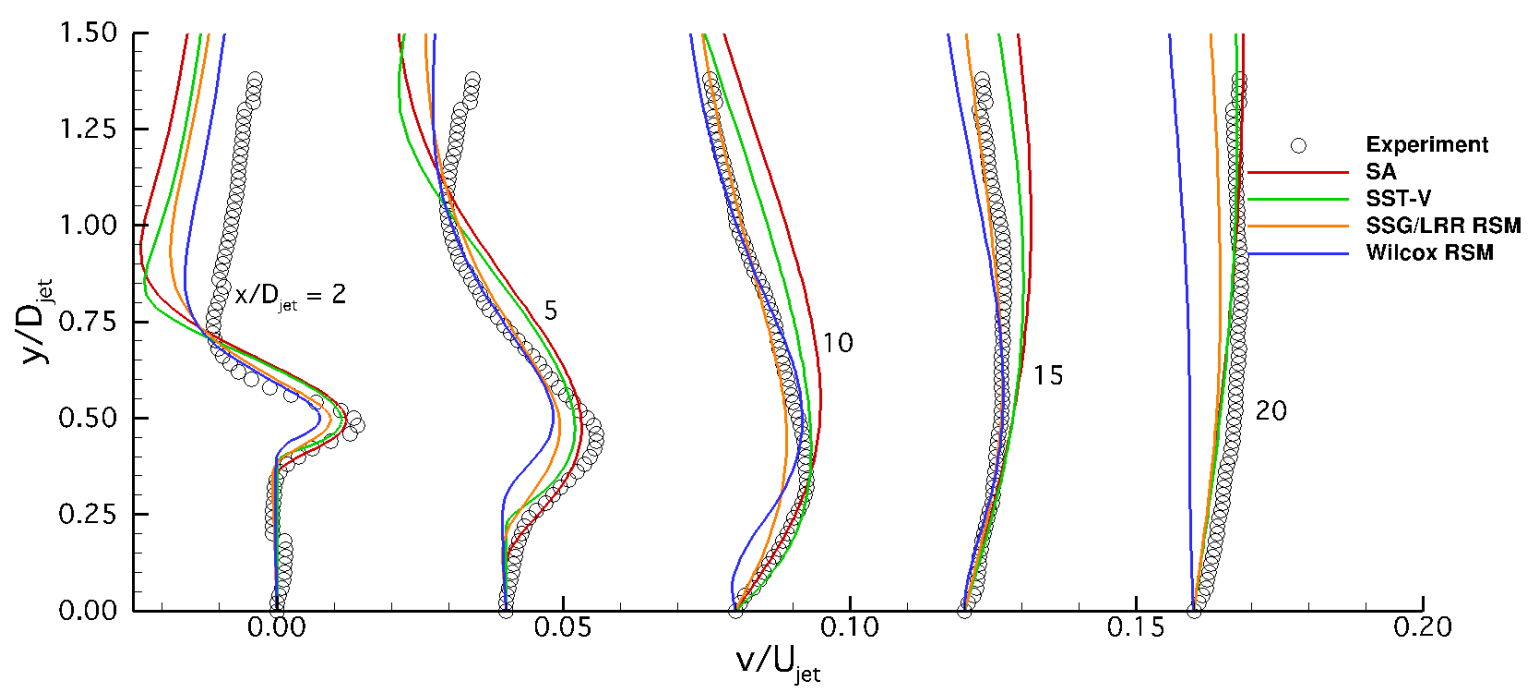

Figure 15.-Set point 7. Radial $y$-velocity profiles. (Subsequent profiles shifted by $u / U_{\text {jet }}=0.04$.)

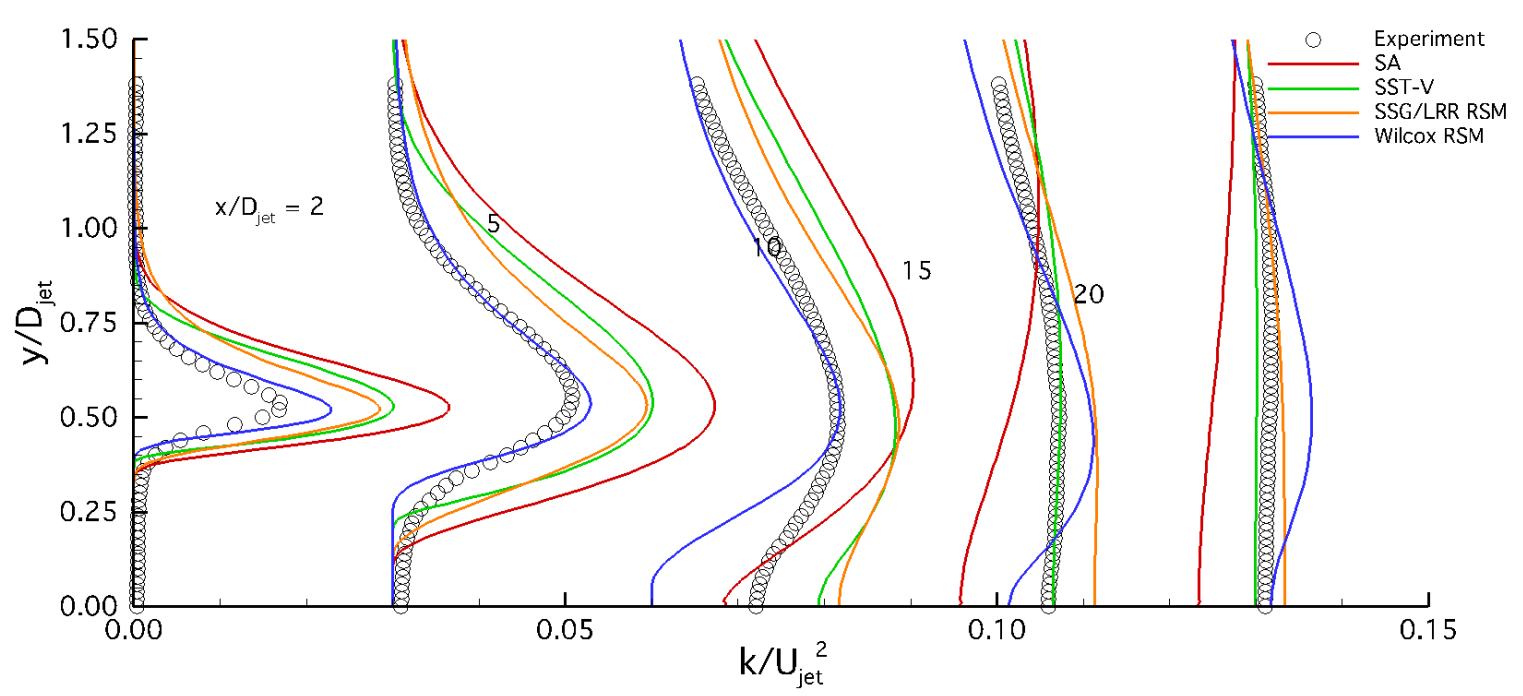

Figure 16. - Set point 7. Radial profiles of turbulent kinetic energy. (Subsequent profiles shifted by $k / \cup_{\text {jet }}{ }^{2}=0.03$.) 

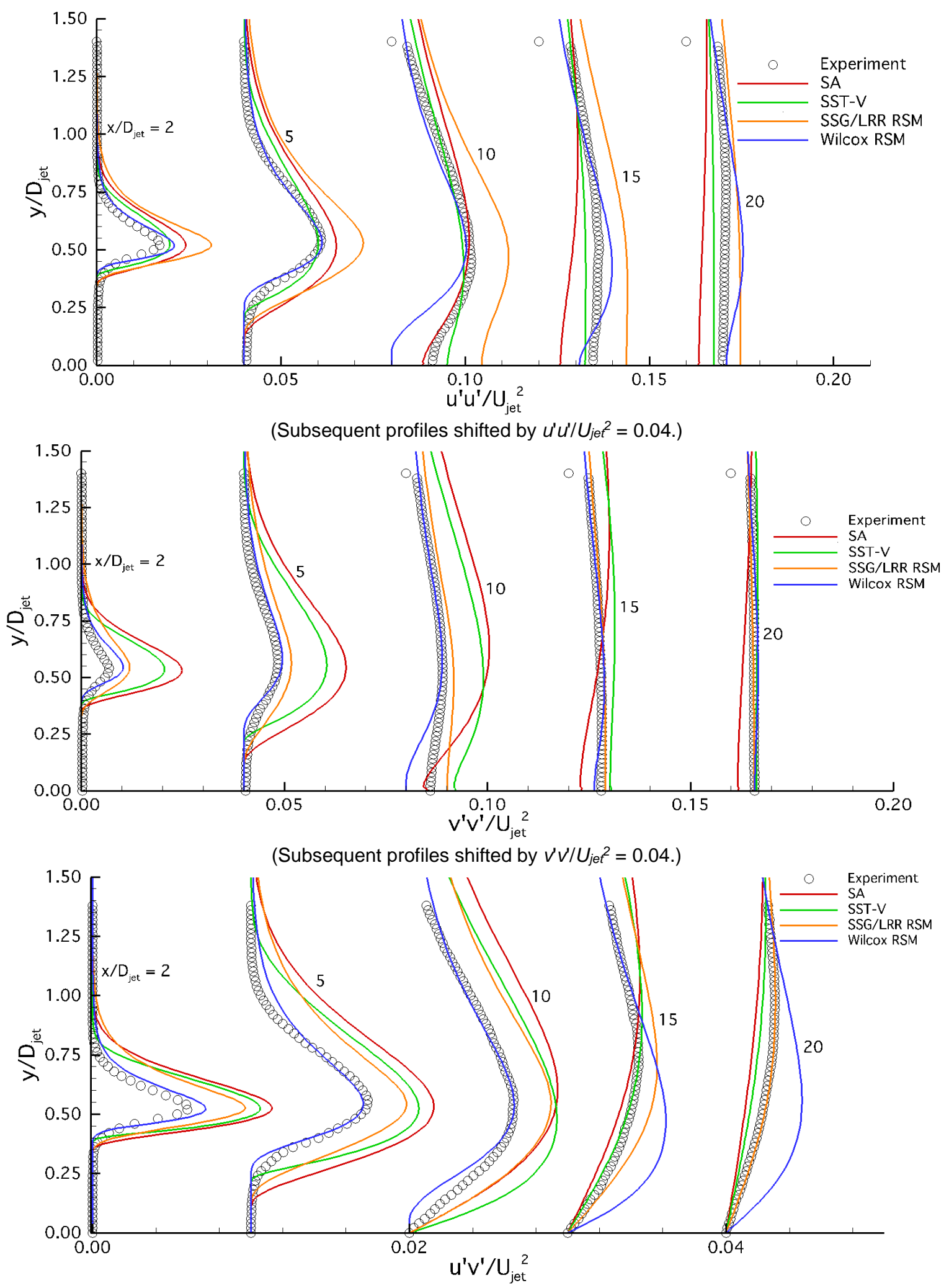

(Subsequent profiles shifted by $u^{\prime} v^{\prime} / U_{j e t}{ }^{2}=0.01$.)

Figure 17. - Set point 7. Radial profiles of the turbulent shear stress components, $u^{\prime} u^{\prime}, v^{\prime} v^{\prime}$, and $u^{\prime} v^{\prime}$. 


\subsection{Conclusion}

Two full second-moment Reynolds stress turbulence models available in the FUN3D code, the Wilcox and the SSG/LRR, were evaluated for axisymmetric jet flow at three conditions: subsonic unheated, subsonic heated, and near sonic unheated. The Reynolds stress model results were compared with results from the commonly used SA and SST-V eddy viscosity models. The flow quantities examined were centerline profiles of the axial velocity and turbulent kinetic energy, and radial profiles of the axial and radial velocity, turbulent kinetic energy and the $u^{\prime} u^{\prime}, v^{\prime} v^{\prime}$, and $u^{\prime} v^{\prime}$ components of the turbulent shear stress. For the centerline axial velocity profile, the SSG/LRR RSM did well for all cases at predicting the potential core length and the downstream mixing. The SA model also did well at predicting the potential core length, however it over-predicted the downstream mixing. The SST-V and the Wilcox RSM over-predicted the potential core lengths in all cases, with the Wilcox RSM significantly overpredicting the core length and under-mixing the downstream flow. Centerline profiles of turbulent kinetic energy exhibited significant scatter between the models, though the profiles predicted by the RSMs both showed similar trends to those of the experiment with the addition of heat to the subsonic nozzle; the peak turbulent kinetic energy increased slightly and the distance of the peak from the jet exit decreased. The turbulent kinetic energy centerline profiles predicted by the eddy viscosity models did not change from the unheated case. The experiment showed that increasing the jet velocity from subsonic to near sonic caused the location of the peak turbulent kinetic energy to move downstream by 2 jet exit diameters compared to the subsonic unheated case, and the value increased slightly. The SA model profile did not change appreciably from the unheated case, and the SST-V, SSG/LRR RSM and Wilcox-RSM had peak values which moved down significantly less than that of the experiment. The radial profiles of velocity and turbulent stresses indicated that the RSMs showed more similarity to experiments.

Of the turbulence models examined in this study, only the SSG/LRR RSM and the SST-V model would be recommended for future jet mixing computations. The SA model would not typically be used because it tends to over-predict the mixing and it does not directly calculate the turbulent kinetic energy or turbulent stresses and therefore does not have the mechanisms to compute the details of the mixing. The Wilcox RSM greatly over-predicted the potential core length for the three flow conditions examined in the study and therefore would not be a likely choice for future jet mixing computations.

Overall, the SSG/LRR RSM is a viable choice for future jet mixing computations. It showed benefits at predicting the jet potential core length and mixing, and the radial components of velocity and turbulence in the potential core. Since it computes all six components of the Reynolds stress, it provides a more accurate distribution of the turbulence quantities to be used for jet noise predictions than traditional eddy viscosity models, yet is a more a practical tool than current LES methods when a timely solution is needed.

\section{References}

1. J. Slotnick, A. Khodadoust, J. Alonso, D. Darmofal, W. Gropp, E. Lurie and D. Marvipilis, "CFD Vision 2030 Study: A Path to Revolutionary Computational Aerosciences," NASA/CR—2014218178, March 2014.

2. H. Choi and P. Moin, "Grid-Point Requirements for Large Eddy Simulation: Chapman's Estimates Revisited," Physics of Fluids, vol. 24, no. 1, pp. 11702-5, 2012.

3. P.R. Spalart and S.R. Allmaras, "A One-Equation Turbulence Model for Aerodynamic Flows," Recherche Aerospatiale, no. 1, pp. 5-21, 1994.

4. F.R. Menter, "Two-Equation Eddy-Viscosity Turbulence Models for Engineering Applications," AIAA Journal, vol. 32, no. 8, pp. 1598-1605, August 1994. 
5. D.C. Wilcox, Turbulence Modeling for CFD, 3rd Edition, DCW Industries, November, 2006.

6. R.-D. Cecora, R. Radespiel, B. Eisfeld and A. Probst, "A Differential Reynolds-Stress Modeling for Aeronautics," AIAA Journal, vol. 53, no. 3, pp. 739-755, 2015.

7. J.C. Dudek and J.-R. Carlson, "Evaluation of Full Reynolds Stress Turbulence Models in FUN3D," AIAA 2017-0541, January 2017.

8. J. Bridges and M.P. Wernet, "The NASA Subsonic Jet Particle Image Velocimetry (PIV) Dataset," NASA/TM-2011-216807, November 2011.

9. R.T. Biedron, J.R. Carlson, M.M. Derlaga, P.A. Gnoffo, D.P. Hammond, W.T. Jones, B. Kleb, E. Lee-Rausch, E.J. Nielsen, C. Rumsey, C.L. Rumsey, J.L. Thomas and W.A. Wood, "FUN3D Manual: 12.9," NASA/TM-2016-219012, February 2016.

10. C.L. Rumsey, "Recent Developments on the Turbulence Modeling Resource Website," AIAA 20152927, June 2015.

11. B. Eisfeld, C. Rumsey and V. Togiti, "Second-Moment RANS Model Verification and Validation using the Turbulence Modeling Resource Website (Invited)," AIAA Paper 2015-2924, June 2015.

12. B.E. Launder, G.J. Reece and W. Rodi, "Progress in the Development of a Reynolds-Stress Turbulence Closure," Journal of Fluid Mechanics, vol. 68, no. 3, pp. 537-566, April 1975.

13. C.G. Speziale, S. Sarkar and T.B. Gatski, "Modelling the Pressure-Strain Correlation of Turbulence -An Invariant Dynamical Systems Approach," Journal of Fluid Mechanics, vol. 227, pp. 245-272, June 1991.

14. B.J. Daly and F.H. Harlow, "Transport Equations of Turbulence," Physics of Fluids, vol. 13, pp. 2634-2649, 1970.

15. B. Eisfeld, "Implementation of Reynolds Stress Models into the DLR-FLOWer Code," Institutsbericht, DLR-IB 124-2004/31, Institute of Aerodynamics and Flow Technology, Branschweig, ISSN 1614-7790, 2004.

16. B.R.C.T.V. Eisfeld, "Verification and Validation of a Second-Moment-Closure Model," AIAA Journal, vol. 54, no. 5, pp. 1524-1540, May 2016.

17. C. Nelson and G. Power, "CHSSI Project CFD-7 - The NPARC Alliance Flow Simulation System," AIAA Paper 2001-0594, January 2001.

18. D.A. Yoder, "Wind-US Users Guide Version 4.0," NASA/TM-2016-219145, September 2016.

19. S.L. Krist, R.T. Biedron and C.L. Rumsey, "CFL3D User's Manual (Version 5)," NASA/TM-1998208444, June 1998.

20. "Pointwise, Inc.," URL: www.pointwise.com.

21. V.N. Vatsa, M.H. Carpenter and D.P. Lockard, "Re-evaluation of an Optimized Second Order Backward Difference (BDF2OPT) Scheme for Unsteady Flow Applications," AIAA Paper 20100122, January 2010.

22. G.D. van Albada, B. Van Leer and W. W. Roberts, "A Comparitive Study of Computational Methods in Cosmic Gas Dynamics," Astronomy \& Astrophysics, vol. 108, p. 76, 1982.

23. J.-R. Carslon, Private correspondance, 2016.

24. R.T. Biedron, J.-R. Carlson, J.G.P.A. Derlaga, D.P. Hammond, W.T. Jones, E.M. Lee-Rausch, E.J. Nielsen, J.A. Park, C.L. Rumsey, J.L. Thomas and W.A. Wood, "FUN3D Manual 13.2," August 2017.

25. N.J. Georgiadis, D.A. Yoder and W.A. Engblom, "Evaluation of Modified Two-Equation Turbulence Models for Jet Flow Predictions," AIAA Journal, vol. 44, no. 12, pp. 3107-3114, 2006. 

